\title{
An Electroweak Enigma: Hyperon Radiative Decays
}

\author{
E. Jastrzembski, J. Lach, J. Marriner \\ Ferimilab \\ Batavia, Illinois 60510 \\ V. Golovtsov, A. Krivshich, V. Schegelsky, N. Smirnov, \\ N. Terentyev, L. Uvarov, A. Vorobyov \\ Leningrad Nuclear Physics Institute \\ Leningrad, USSR \\ E. Mccliment, C. Newsom, E. Norbeck \\ University of lowa \\ lowa City, lowa 52242 \\ P. S. Cooper \\ Yale University \\ New Haven, Connecticut 06511
}




\section{An Electroweak Enigma: Hyperon Radiative Decays}

Goals. The main thrust of this experiment will be to measure the asymmetry parameter for the electroweak decay $\Sigma^{+} \rightarrow p \gamma$ and verify its branching ratio. As a secondary goal we will measure, or set new upper limits for, the branching ratio of the electroweak decay $\Xi^{-} \rightarrow \Sigma^{-} \gamma$. Since the $\Xi^{-}$are expected to be polarized, information on the asymmetry parameter may also be available.

Motiviation. The reactions $\Sigma^{+} \rightarrow p \gamma$ and $\Xi^{-} \rightarrow \Sigma^{-} \gamma$ represent a class of baryon radiative decays which are strangeness changing, hence requiring both weak and electromagnetic contributions. This feature allows a unique opportunity to test our understanding of the interplay of weak and electromagnetic theory as applied to the underlying quark structure of the baryons. This realization is not new; theorists have urged their experimental colleagues to investigate the properties of these decays for decades. However these are very difficult experiments and the statistical quality and systematic uncertainties of present data does not lend itself to hard conclusions. Whether these few experiments have obfuscated the issue or are indications of fundamental misunderstandings is unclear. What is clear is that modern high energy, high intensity polarized hyperon beams have shown themselves to be unique instruments ideally suited to the study of such rare decay processes.

The hyperon electroweak decays include the following reactions. The branching ratios and values of the decay asymmetry perameter, $\alpha_{\gamma}$, are from the current Particle Data Group(1984) compilation.

$\begin{array}{lll} & \text { Branching Ratio } & \alpha \gamma \\ \Sigma^{+} \rightarrow p \gamma & (1.20 \pm 0.13) \times 10^{-3} & -0.72 \pm 0.29 \\ \Xi^{-} \rightarrow \Sigma^{-} \gamma & <1.2 \times 10^{-3} & - \\ \Xi^{\circ} \rightarrow \Lambda \gamma & (5.0 \pm 5.0) \times 10^{-3} & - \\ \Xi^{\circ} \rightarrow \Sigma^{\circ} \gamma & <7.0 \times 10^{-3} & - \\ \Omega^{-} \rightarrow \Xi^{-} \gamma & <3.1 \times 10^{-3} & - \\ \Lambda \rightarrow n \gamma & - & -\end{array}$

The paucity of experimental data relevant to these decays is striking. 
Only for $\Sigma^{+} \rightarrow p \gamma$ is there any information on the spin structure of the decay. Note that we do not include the reaction $\Sigma^{\circ} \rightarrow \wedge \gamma$ since this an electromagnetic decay not involving the weak interactions.

We point out that the mechanism of these radiative decays have common features with two other problems of current interest. These involve the $\Delta I=1 / 2$ rule and the $\epsilon / \epsilon^{\prime}$ ratio in kaon decay. The non-leptonic Hamiltonians for these three problems are closely related. Insights, either experimental or theoretical, gleaned from one of these problems may be extremely relevant to the understanding of the others.

We propose to look at the decay of polarized $\Sigma^{+} \rightarrow p \gamma$ and $\Xi^{-} \rightarrow \Sigma^{-} \gamma$ with a modern apparatus and measure both their branching ratios and the distribution of their decay products with respect to the initial hyperon polarization direction. We will have statistics of $10^{4}-10^{5}$ events, at least two orders of magnitude larger than all of the previous experments for the $\Sigma^{+}$decay. Our systematic and statistical uncertainties will be such as to produce unambiguous results.

The flux and polarization of $\Sigma^{+}$and $\Xi^{-}$hyperons have not as yet been measured at Tevatron energies. Not only is this measurement essential to our determination of the $\Sigma^{+}$asymmetry parameter, but it is of inherent interest in itself.

Theoretical Background The transition matrix for the general electroweak decay

$$
B(D) \rightarrow B^{\prime}\left(p^{\prime}\right)+\gamma(Q)
$$

where $B$ and $B^{\prime}$ refer to the initial and final state baryon can be taken from Marshak

$$
T \sim \epsilon_{v} \bar{u}\left(p^{\prime}\right)\left(a+b \gamma_{5}\right) \sigma_{\mu v} Q_{\mu} u(p)
$$

where $D, D^{\prime}$ and $Q$ are the 4-momenta of the respective particles and $\epsilon_{v}$ is the polarization vector of the photon. We can write the branching ratio (BR) as

$$
B R \sim|a|^{2}+|b|^{2}
$$

while the asymmetry parameter $\alpha_{\gamma}$ is given by 


$$
\alpha \gamma=2 \operatorname{Re} a^{*} b /\left(|a|^{2}+|b|^{2}\right)
$$

Knowing $\alpha \gamma$, we candetermine the relative magnitude of the odd (a) and even (b) parity amplitudes. We can measure $\alpha_{\gamma}$ from the center of mass angular distribution of the decay baryons relative to the initial hyperon spin direction

$$
d N(\cos \theta)=N_{0}\left(1+\alpha_{\gamma} \hat{\sigma}_{B^{\prime}} \cdot \hat{P}_{B^{\prime}}\right) d \Omega
$$

where $\hat{\sigma}_{B}$ and $\hat{P}_{B}$, are unit vectors in the direction of the initial hyperon polarization and the momentum of the decay baryon respectively.

Early work to describe the $\Sigma^{+} \rightarrow p \gamma$ decay centered on "Dole models" but these had difficulty predicting both the measured rate and the asymmetry parameter [Graham and Pakvasa (1965), Farrar (1971), Holstein (1971), Gavroglu and Gottlieb (1974)].

The constituent quark model has worked well in giving us an understanding of the baryon magnetic moments ( $20 \%)$. It is instructive to consider the radiative decays in the same context. The basic diagram involves the conversionof an s quark to a d quark with the emission of a photon.

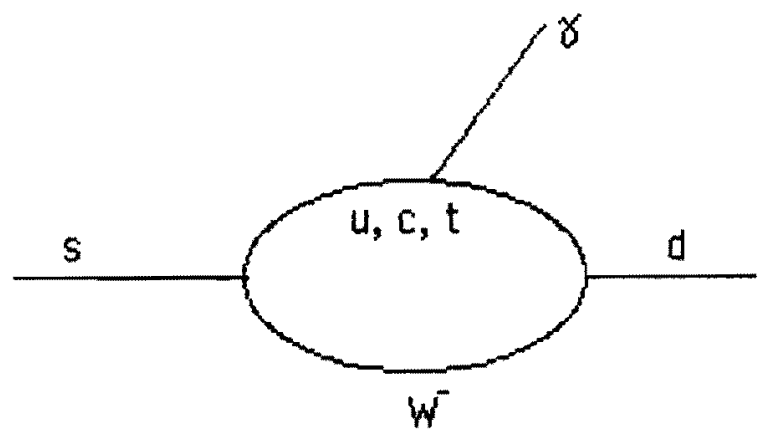

In an illuminating historical digression at the beginning of Kogan and Shifman (1983), they point out that such diagrams lead to branching ratios which are much too small for the $\Sigma^{+} \rightarrow p \gamma$ decay. 
Gilman and Wise (1979), without looking at a particular model, attempt to see if a consistent picture of these electroweak decays can be made from the general assumption that they all arise from a strange quark decaying into a down quark with the emission of a photon. Gliman and Wise normalize these diagrams to the measured rate for $\Sigma^{+} \rightarrow p \gamma$ and with this normalization they are then able to predict the other decay rates.

\section{Predicted}

$$
\begin{aligned}
& \Lambda \rightarrow n \gamma \\
& \Xi^{-} \rightarrow \Sigma^{-} \gamma \\
& \Xi^{\circ} \rightarrow \Sigma^{\circ} \gamma \\
& \Xi^{\circ} \rightarrow \Lambda \gamma \\
& \Omega^{-} \rightarrow \Xi^{-} \gamma
\end{aligned}
$$

$2.2 \times 10^{-2}$

$1.1 \times 10^{-2}$

$9.1 \times 10^{-3}$

$4.0 \times 10^{-3}$

$4.1 \times 10^{-2}$

\section{Measured}

$<1.2 \times 10^{-3}$

$<7.0 \times 10^{-3}$

$<(5.0 \pm 5.0) \times 10^{-3}$

$<3.1 \times 10^{-3}$

It is clear that the experimental upper limits of the $\Xi^{-}$and $\Omega^{-}$electroweak branching ratios are not consistent with that of the $\Sigma^{+}$in this picture.

We can look at the effects of the simplest four quark diagram

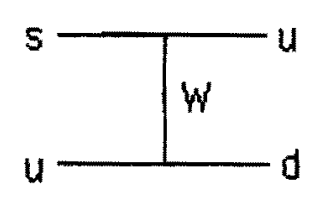

As applied to the decay $\Sigma^{+} \rightarrow \mathrm{pr}$

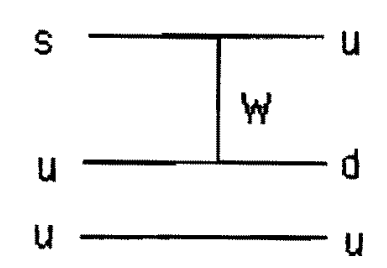

where the photon could come from any of the quark lines. It was emphasized by (e.g. Rudaz, 1984) that such diagram could give substantial contributions to the $\Sigma^{+} \rightarrow p \gamma$ rate but could not contribute to $\Xi^{-} \rightarrow \Sigma^{-} \gamma$ or $\Omega^{-} \rightarrow \Xi^{-} \gamma$ since neither the initial $\Xi^{-}$or $\Omega^{-}$contain a valence $u$ quark.

There is a class of diagrams [Gilman and Hagelin (1979). Eeg (1982), 
Shifman et al., (1977)] that are believed to play an important role in hyperon non-leptonic decays ( $\Delta \mathrm{l}=1 / 2$ and octet enhancement) and in neutral kaon decays $\left(\epsilon / \epsilon^{\prime}\right)$. These are the penguin diagrams which have one or more gluon lines connecting the quarks

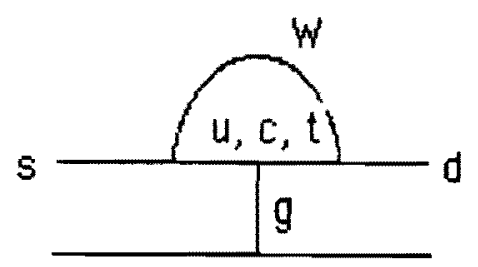

Our electroweak decays would be of this variety but with a photon being emitted from one of the quark lines. A photon emitted from an external quark line is directly cornected with the diagrams relevant to $\Delta l=1 / 2$ and $\epsilon / \epsilon$. A photon emitted from the internal quark loop would be unique to the radiative decays and might allow new insights into the interplay of the weak and electromagnetic interactions. It seems the $\Xi^{-}$and $\Omega^{-}$radiative decays may be a third way to probe these penguin contributions although qualitative computations give branching ratios which are too small (Eeg, 1982).

Lower bounds to these branching ratios can be derived from unitarity limits as was first pointed out by Kawaguchi and Nishijima (1956), Iso and Kawaguchi (1956) and more recently by Kogan and Shifman (1983). The following diagram illustrates this for $\Xi^{-} \rightarrow \Sigma^{-} \gamma$

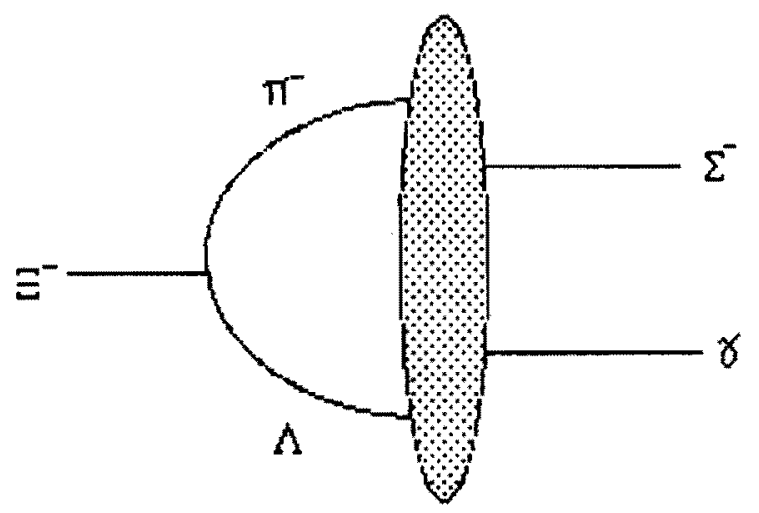


This can be broken up into two parts. There is a part which is given by the known $\Xi^{-}$two body decay rates and a photoproduction part which can be estimated. This kind of estimation has been attempted by a number of authors. Kogan and Shifman (1983) estimate also the real part for these reactions so they are able to compute a branching ratio.

Branching Ratio

$\begin{array}{ll}\Xi^{-} \rightarrow \Sigma^{-} \gamma & 1.7 \times 10^{-4} \\ \Omega^{-} \rightarrow \Xi^{-} \gamma & (1.0-1.5) \times 10^{-5} \\ \Sigma^{+} \rightarrow p \gamma & \sim 10^{-3}\end{array}$

Symmetry principles were recognized at an early date as impossing important constraints on the radiative decays. Hara in 1964 showed that the current-current form of the weak interaction combined with the assumption of left handed currents, $C P$ invariance, and $U$ spin symmetry required the asymmetry parameter in these reactions to be zero in the $\mathrm{SU}_{3}$ limit.

It was thought that these reactions would be likely places to search for right handed currents (Ahmed and Ross, 1975); however more recent work (Shifman et al, 1978) indicate that these effects should be unimportant. If a large and negative value of $\alpha_{\gamma}$ were to be established, as present experiments indicate, we would have to rethink the role of the symimetry principles assumed by Hara.

In the recent hyperon workshop. Rudaz (1984) reminded us that if we relax the requirement of $U$ spin symmetry we can write $\alpha_{\gamma}$ for the decay $\Sigma^{+} \rightarrow$ pr as

$$
\begin{aligned}
\alpha_{\gamma} & =\left(m_{s}^{2}-m_{d}^{2}\right) /\left(m_{s}^{2}+m_{d}^{2}\right) \\
& \sim+0.5
\end{aligned}
$$

This has the opposite sign from the present experimental data. Numerous attempts have been made to reconcile the measured large negative value of $\alpha_{\gamma}$ but none are entirely convincingand the problem still remains an enigma. 
Current Experimental Status. The current experimental situation has been reviewed by Bourquin and Repellin (1984). We note that almost all of the relevant data on the decay $\Sigma^{+} \rightarrow p \gamma$ comes from two bubble chamber experiments: Gershwin et al (1969) and Manz et al (1980). We summarize these experiments below

\section{Gershwin et al Manz et al}

Detector

Number of events

$\Sigma^{+} \rightarrow p \gamma / \Sigma^{+} \rightarrow p \pi^{\circ}\left(\times 10^{3}\right)$

$\alpha \gamma$
LBL 25" HBC

$31\left(61\right.$ for $\left.\alpha_{\gamma}\right) \quad 35$

$2.76 \pm 0.51$

$-1.03-0.42^{+0.52}-0.53-0.36+0.38$

The asymmetry and rate as given by the Particle Data Group are

$$
\begin{aligned}
\alpha \gamma & =-0.72 \pm 0.29 \\
\Sigma^{+} \rightarrow p \gamma / \text { all } & =(1.20 \pm 0.13) \times 10^{-3}
\end{aligned}
$$

One notes that the two measurements are consistent althought the individual errors on $\alpha_{\gamma}$ are quite large. The average for $\alpha_{\gamma}$ is almost three standard deviations from zero. However, this sample contains only about one hundred events.

\section{IHE EXPERIMENI}

Design Strategy. The first priority of this proposal is to study the decay $\Sigma^{+} \rightarrow p \gamma$ with the goal of a definitive measurement of $\alpha \gamma$ and a confirmation of the branching ratio. As a secondary priority we plan to study the decay $\Xi^{-} \rightarrow \Sigma^{-} \gamma$. The only experimental information available on this decay is an upper limit on the branching ratio. As noted above, its branching ratio is predicted from unitarity arguments. We will measure or set new upper limits to the branching ratio. The statistical precision of $\alpha \gamma$ for $\Xi^{-} \rightarrow \Sigma^{-} \gamma$ will depend on the value of the branching ratio. 
These two reactions has similarities which we hope to exploit. Both parent particles ( $\Sigma^{+}$and $\Xi^{-}$) constitute a 1-2\% beam fraction $(350 \mathrm{GeV} / \mathrm{C}$ ) at $10 \mathrm{~m}$ from their production target. Both decay to a fast forward charged baryon and a single photon. High resolution spatial dectectors will measure the kinking charged trajectories and a spectrometer determines the secondary baryon momentum. A novel application of a transition radiation detector (TRD) which determines the photon conversionpoint to high accuracy is followed by an array of lead glass blocks which determines its energy.

The major background in the $\Sigma^{+}$case will come from the much more copious decay $\Sigma^{+} \rightarrow p \pi^{\circ}(\sim 52 \%)$. The $\Sigma^{+} \rightarrow p \gamma$ and $\Sigma^{+} \rightarrow p \pi^{\circ}$ decays have similar final state particles and kinematics, thus providing a challenge to the trigger. We will use the fact that the background $\Sigma^{+} \rightarrow D \pi^{\circ}$ reaction will give us two photons and reject a substantial number ( $\sim 50 \%)$ on that basis. A fast processor will require coplanarity of the three tracks of the decay $\Sigma^{+} \rightarrow p \gamma$ thus reducing the background by about another order of magnitude. Note that our desired decay and its background occur at a relative rate of $\sim 1: 500$, and a trigger which reduces this by a factor of ten would be acceptable.

Off line we have sufficient precision in the measurement of the $\Sigma^{+}$and the proton momentum vectors to distinquish the two reactions by their characteristic missing mass ( $\gamma$ versus $\pi^{\circ}$ ). A measurement of the $\gamma$ angle and energy will then provide enough redundancy to make the identification unequivocal.

The clean identification of the reaction $\Xi^{-} \rightarrow \Sigma^{-} \gamma$ probably requires that we measure the magnitude of the $\Sigma^{-}$momentum as well as its direction. This requirement, through its constraint on the $\Sigma^{-}$lifetime of the accepted events, reduces our acceptance for this decay by about an order of magnitude. Depending on the nature of the background, it might be sufficient to measure just the photonenergy, not its direction, using the two lead glass arrays which restores a factor of $\sim 2$ in acceptance. This may be feasible because there is no copiously produced state with similar kinematics as there is with the $\Sigma^{+}$. The only serious source of background is from the decay chain: $\Xi^{-} \rightarrow \Lambda \pi^{-}, \Lambda \rightarrow n \pi^{\circ}, \pi^{\circ} \rightarrow \gamma \gamma$. This should be easily distinguishable off line.

It is interesting to note that contained in the same trigger as the $\Xi^{-} \rightarrow \Sigma^{-} \gamma$ events will be a small sample of $\Omega^{-} \rightarrow \Xi^{-} \gamma$ decays. These may be 
separable from the $\Xi^{-} \rightarrow \Sigma^{-} \gamma$ sample thus providing an estimate of the branching ratio.

Experimental Configuration. The experimental configuration is depicted in Figure 1 . Shown is the Proton Center hyperon targetting magnet with a curved channel which would allow beams of at least 350 $\mathrm{GeV} / \mathrm{C}$. The identical channel used in E497 and E715 would be acceptable for this experiment.

Directly downstream of the hyperon targetting magnet are a set of two silicon strip detector stations (SD1, SD2) which would measure the position and angle of the beam particles. These detectors need only have an active area of about $1 \mathrm{~cm}^{2}$ each and measure the position of beam tracks to $10 \mu \mathrm{m}$ in each coordinate. Resolutions are given in standard deviations $(\sigma)$ unless noted. Althought we require high spatial resolution from these detectors, we are dealing with only a single track traversing them.

We take as the origin of our coordinate system the exit aperture of the hyperon targeting magnet. Note that $x$ is in the bend (horizontal) plane of the hyperon targetting magnet, $z$ is along the beam direction. and $y$ is vertical (Figure 1).

At the end of the $12 \mathrm{~m}$ decay region, a proportional wire chamber ( $P W C 1$ ) determines the $x$ and $y$ coordinated of the decay proton trajectory. This chamber station need only have an active area of $5 \times 5 \mathrm{~cm}^{2}$ and should provide a spatial resolution of $\sim 200 \mu \mathrm{m}$ in each coordinate. As an option we are also considering a silicon strip detector which would give improved spatial resolution ( $\sim 50 \mu \mathrm{m})$.

About 20 m downstream of PWCl we place a detector to intercept the $\gamma$ from the hyperon radiative decay. This detector consists of two parts. The $\gamma$ spatial coordinate will be measured by a transition radiation detector placed just downstream of 1 radiation length $\left(L_{r}\right)$ Pb converter. The energy of the $\gamma$ will be measured by a lead glass calorimeter (LGI) just downstream of the TRD chamber.

We expect that the position of the $\gamma$ shower can be located to about $0.5 \mathrm{~mm}$ by the TRD chambers and that it will be an innovative application of this device. We will discuss this further in a subsequent section.

We estimate that the $\gamma$ energy can be measured to an accuracy of $\sim 12 \% / \sqrt{ } \mathrm{E}_{\gamma}$ (as achieved in E715) where $\mathrm{E}_{\gamma}$ is the $\gamma$ energy measured in $\mathrm{GeV}$. The spatial resolution of the lead glass array is not of major 
importance since it is expected that of the TRD would have adequate. The off line rejection of some $\pi^{\circ}$ events in which both photons convert in the lead glass could be facilitated if the blocks had adequate segmentation.

The lead glass array and the PD converter would be of modest size covering an area of $\sim 80 \times 80 \mathrm{~cm}^{2}$. A hole of $\sim 10 \times 10 \mathrm{~cm}^{2}$ would allow beam and decay protons to pass through. A second similar $\gamma$ detector (LG2) intercepts those photons that pass through the beam hole of LG1 and is located much further downstream (Figure 1).

SD1 and SD2 measure the incident hyperon angle, PWC1 and PWC2 measure the decay proton angle. Each of these measurements is done to a precision of $\sim 10 \mu \mathrm{rad}$. Another set of chambers, PWC3 and PWC4 measure the proton angle after the set of three analysing magnets. We assume each of these magnets is a BM109 type magnet operating at $18 \mathrm{KG}$ and acting together giving a total $P_{t}$ deflection of $2.9 \mathrm{GeV} / \mathrm{c}$. Note that we require only modest vertical apertures from these magnets ( 8 inches) so that a magnetic field of $18 \mathrm{kG}$ is reasonable. We summarize the parameters of the detectors.

$\begin{array}{llll}\text { Detector } & \text { Z Position } & \text { Size } & \text { Resolution } \\ \text { SD1 } & 1 \mathrm{~m} & 0.6 \times 1.0 \mathrm{~cm}^{2} & 10 \mu \mathrm{m} \\ \text { SD2 } & 4 & 1.3 \times 1.3 & 10 \\ \text { PWC1 } & 16 & 5 . \times 5 . & 200 \\ \text { TRD1 } & 36 & 60 \times 60 . & 500 \text { (for } \gamma) \\ \text { PbG1 } & 39 . & 80 \times 80 . & \\ \text { PWC2 } & 40 & 10 \times 10 . & 200 \\ \text { PWC3 } & 46 & 15 . \times 10 . & 200 \\ \text { PWC4 } & 101 & 50 . \times 25 . & 300 \\ \text { PbG2 } & 101.5 & 50 \times 50 . & \end{array}$

Although PWC1 has a modest sized active area, only a minimum of material can be tolerated out to an area of $25 \times 25 \mathrm{~cm}^{2}$ since the photons must traverse this region. Scintillation counters necessary for a straight forward trigger are also indicated in Figure 1. Helium bags will be needed in order to achieve goodmissing mass resolution.

The above geometry is for the $\Sigma^{+} \rightarrow p \gamma$ part of the experiment. For the decay $\Xi^{-} \rightarrow \Sigma^{-} \gamma$. we would use the same geometry (all magnetic fields are reversed) except that the separation between some of the PWC's would be 
decreased. In order to reduce the backgrounds from umwanted decays of beam $\Sigma^{-}$and $\Xi^{-}$. we may install additional scintillation counters to reject low momentum charged particles from their decays. We feel that this and a combination of on line cuts can reduce backgrounds to an acceptable level.

Beam And Polarization. We propose to use the Proton Center charged hyperon beam appropriately modified to target $800 \mathrm{GeV}$ protons and transport a positive or negative secondary beam of approximately 350 $\mathrm{GeV} / \mathrm{C}$. Our experience with E497 where we used a similar but lower momentum positive beam and made a precision measurement of the $\Sigma^{+}$ magnetic moment (Ankenbrandt et al 1983) assures us that this is practical.

Charged hyperon fluxes have not been measured above an incident proton energy of $400 \mathrm{GeV}$. However there exist rather detailed measurements at $400 \mathrm{GeV}$ from Fermilab E497 (Cardello et al 1984). A recent extrapolation of these results to $800 \mathrm{GeV}$ (Jastrzembski 1984) is shown in Figure 2 for positive particle fluxes at a $P_{t}=1.25 \mathrm{GeV} / \mathrm{c}$. Figure 2 shows the postive beam composition at a distance of $10 \mathrm{~m}$ from the production target as a function of secondarymomentum. We note that the $\Sigma^{+}$fraction is not a very strong function of the secondary momentum (hence Feynman $x$ ) above about $\sim 300 \mathrm{GeV} / \mathrm{c}$. The $\Sigma^{+}$fraction of the beam is about $1.5 \%$ at $350 \mathrm{GeV} / \mathrm{c}$.

Figure 3 displays the $\Sigma^{+}$polarization measurements. This figure is taken from Ankenbrandt et al (1983) and includes data from Fermilab E497 and E620 (Wilkinson et al 1981). All were produced by $400 \mathrm{GeV}$ protons. One notes that for $0.50<P_{t}<1.50 \mathrm{GeV} / C$, the polarization is about 20\%. Although there are no measurements of the $\Sigma^{+}$polarization at $800 \mathrm{GeV}$, there does not appear to be a strong energy dependence to the hyperon polarizations (Heller 1984). However there are some indications that hyperon polarizations increase with increasing $x$ (Heller 1984). The desire to have larger polarization by working at larger $x$ is in opposition to the need of good mass resolution which decreases with higher $\Sigma^{+}$ momentum.

For the $\Sigma^{+}$run we will tentatively choose a working momentum of 350 $\mathrm{GeV} / \mathrm{C}$ at a $P_{\mathrm{t}}=1.25 \mathrm{GeV} / \mathrm{C}$. This is at a somewhat lower Feynman $x$ value that used for E497 $\Sigma^{+}$magnetic moment measurement, hence probably 
slightly lower polarization. Going from a copper target (as used in E497) to one of beryllium should compensate for the loss of polarization due to the smaller $x$. We expect a polarization of about $20 \%$ which we would conf irm with a sample of $\Sigma^{+} \rightarrow p \pi^{\circ}$ events.

We propose to use the same angle changing magnets as used for E497 which allowed a $\pm 7.5 \mathrm{mrad}$ deflection of the incident $400 \mathrm{GeV}$ proton beam. At $800 \mathrm{GeV}$ this would allow an angular deflection of $\pm 3.75 \mathrm{mrad}$, thus with a $350 \mathrm{GeV} / \mathrm{C}$ secondary beam providing a maximum $P_{t}=1.3$ GeV/c.

Figure 4 shows the expected negative fluxes at $350 \mathrm{GeV} / \mathrm{c}$ from 800 $\mathrm{GeV}$ incident protons at a $P_{t}=1.25 \mathrm{GeV} / \mathrm{C}$ (Jastrzembski, 1984). Note that the $\Xi^{-}$flux at a distance of $10 \mathrm{~m}$ from the production target is $\sim 2 \%$. Figure 5 (Rameika, 1981) shows the available $\Xi^{-}$polarization data. We expect it to be $\sim 10 \%\left(1 / 2\right.$ that of $\left.\Sigma^{+}\right)$.

Resolution. The results presented here are the results of an extensive set of Monte Carlo computations for $\Sigma^{*} \rightarrow p \gamma$. This decay has the more serious background problem and is the primary goal of this experiment. A precision measurement of both the $\Sigma^{+}$and proton momentum vectors are necessary since it is from them that we construct the missing mass which is our most important off line tool to distinguish between the $\Sigma^{+} \rightarrow p \gamma$ and $\Sigma^{+} \rightarrow p \pi^{\circ}$ decays.

$$
M M^{2}=\left(P_{\Sigma}-P_{D}\right)^{2}
$$

where $P_{\Sigma}$ and $P_{D}$ are the $\Sigma^{+}$and proton 4-vectors. We can estimate this missing mass squared $\left(\mathrm{MM}^{2}\right)$ resolution from the detector parameters tabulated above. The precision of the hyperon momentum determination can be computed from it's position $\left(a_{x}\right)$ and angle $\left(\theta_{x}\right)$ uncertainty as determined by the silicon strip detectors SD1 and SD2 as well as the uncertainty in the production target size $\left(\Delta x_{t}=0.3 \mathrm{~mm}[\sigma]\right)$, the length of the magnetic channel $\left(Z_{m}=7.0 \mathrm{~m}\right)$, and its radius of curvature $(\rho=350 \mathrm{~m})$. in terms of these quantities

$$
\Delta P_{\Sigma} / P_{\Sigma}=\rho / Z_{m}^{2}\left(\Delta x_{t}{ }^{2}+\Delta a_{x}^{2}+Z_{m}^{2} \Delta \theta_{x}^{2}\right)^{1 / 2}
$$


and the uncertainty of the transverse momentum components will be $\Delta \mathrm{P}_{X}=\Delta \mathrm{P}_{y} \sim 1.7 \mathrm{MeV} / \mathrm{C}$ and the uncertainty in $\Delta \mathrm{P}_{\Sigma} / \mathrm{P}_{\Sigma} \sim 0.15 \%$. This combined with the detector position and resolution data in the previous table, allow us to calculate the missing mass squared error for the two decays.

$$
\begin{aligned}
& \sigma_{\mathrm{mm}^{2}}\left(\pi^{\circ}\right)=1.7 \quad 10^{-3}(\mathrm{GeV} / \mathrm{c})^{2} \\
& \sigma_{\mathrm{mm}^{2}}(\gamma)=2.1 \quad 10^{-3}(\mathrm{GeV} / \mathrm{c})^{2}
\end{aligned}
$$

With the assumption that these errors are Gaussian, we compute that the probability of a $\Sigma^{+} \rightarrow p \pi^{\circ}$ event to be mistaken for a $\Sigma^{+} \rightarrow p \gamma$ event is $\sim 10^{-6}$; the probability that the $\pi^{\circ} \mathrm{MM}^{2}$ will be measured to be less than 0.005 $(\mathrm{GeV} / \mathrm{C})^{2}$. This result is encouraging but certainly optimistic since there will likely be non-Gaussian tails to these distributions.

At this juncture we must admit a certain uneasiness about achieving such very good $\Sigma^{+}$momentum resolution. A certain number of off mometum particles originating from interactions in the channel walls will mimic trajectories coming from the target. If we can convince ourselves that this might be a serious background, we would propose to introduce a short $(2-3 \mathrm{~m})$ small aperture $($ a few $\mathrm{cm}$ ) high field ( $45 \mathrm{kG}$ ) magnet just downstream of the hyperon targeting magnet. An additional silicon strip detector between this magnet and the hyperon targeting magnet will serve to reject these particles off line. Since both $\Sigma^{+}$and the $\Xi^{-}$have decay lengths of about $12 \mathrm{~m}$ at $350 \mathrm{GeV} / \mathrm{c}$, the decay losses necessitated by this extra length could be tolerated.

The position of the photon conversion in the TRD and energy as determined by the lead glass also provides important data to help distinguish these two decays. The use of coplanarity as an on line requirement has already been mentioned. In Figure $6 a$ we plot the deviation from coplanarity of the photons from the $\Sigma^{+} \rightarrow D \pi^{\circ}$ decay at their conversionpoint near the TRD. Also indicated is the expected resolution of our on line coplanarity algorithm. From such a figure it is seen that an on line rejection of about 10 can be expected. Of course, in addition to coplanarity, the photon space angle, Figure $6 \mathrm{~b}$, provides an even more demanding constraint. 
Polarization Measurement Using $\Sigma^{+} \rightarrow p \pi^{\circ}$. To deduce $\alpha_{\gamma}$ from the decay angular distribution, we must have an independent measurement of the $\Sigma^{+}$polarization. We plan to do this from a sample of $\Sigma^{+} \rightarrow p \pi^{\circ}$ decays recorded simultaneously with $\Sigma^{+} \rightarrow p \gamma$. A systematic set of polarization measurements in this kinematic region of $\Sigma^{+}$production would be important to the understanding of polarization phenomenology. We propose to do these measurements as part of this experiment. We remark that the $\Sigma^{+} \rightarrow p \pi^{\circ}$ reaction is particularly well suited as an analyser of polarization since the asymmetry parameter for this decay is almost maximal $(\alpha=-0.979)$.

The TRD As a Precision Spatial Gamma Detector. Our experience with E715 has demonstrated that the TRD has come of age. We feel that it is a device whose response is calculable and whose range of applicability is just being realized. In this experiment we propose to use it as a precision spatial gamma detector. We note that when a high energy photon traverses lead converter (thickness of about one radiation lenght), most of the high energy electrons produced follow closely the initial photon direction. Quantatively, $92 \%$ of the electrons are contained in a cone given by

$$
{ }_{\gamma_{e}}=3\left(m_{e} / E_{e}\right)
$$

where $E_{e}$ is the electron energy and $m_{e}$ is its rest mass. Note that since the threshold energy (Figure 7, taken from Denisov, 1984) for an electron in our TRD is $\sim 2.5 \mathrm{GeV} / \mathrm{c}$, this correspondsto an angle of $<0.5 \mathrm{mrad}$. The angle of the TRD $X$-rays from these electons is given by

$$
\theta_{\mathrm{ex}} \approx m_{\mathrm{e}} / \mathrm{E}_{\mathrm{e}}
$$

so that the TRD $x$-rays follow the direction of the initial high energy photon quite closely. This means that a TRD will be mainly sensitive to the fast forward electon component of the electomagnetic shower.

A Monte Carlo program which simulates electromagnetic showers $(\mathrm{N}$. Mokhov, private communication) was used to compute the electron distribution after one radiation length of lead for various energies of photons. In Figure 8 we plot the number of electrons produced with 
photon energies between $10-150 \mathrm{GeV}$. Figure 9 displays the number of detected ionization cluster per TRD chamber as a function of incident photon energy. Note that we plot the response due to produced electrons less than $2.5 \mathrm{GeV}$ and greater than $2.5 \mathrm{GeV}$ separately showing the enhanced response for the high energy electrons. This is characteristic of the cluster counting technique.

The TRD geometry would consist of a one radiation length lead converter (with an appropriate hole for the beam) followed by four ( $2 x$, 2y) TRD modules each with its own polypropylene radiator and Xenon proportional chamber. The photon detection efficiency is determined mainly by the thicknes of the converter ( 60\%).

The chambers would have a wire spacing of $2 \mathrm{~mm}$ (same as for E715) but able to count the number of clusters on each wire. By staggering the chambers by $1 / 2$ of their wire spacing, tilting them about $7^{\circ}$ to the beam and finding the center of gravity of the produced ionization clusters, we estimate that the incident photon can be located to $\sim 0.5 \mathrm{~mm}(\sigma)$ in $\mathrm{x}$ and $y$. Following the TRD would be a lead glass array to determine the energy of the incident photon.

RATES: $\Sigma^{+} \rightarrow$ pr. We expect that the charged Deam will contain $\sim 1.5 \% \Sigma^{+}$particles. The decay $\Sigma^{+} \rightarrow p \gamma$ represents oniy $(1.20 \pm 0.13) \times 10^{-3}$ of all $\Sigma^{+}$decays (Particle Data Group 1984). Assuming the nominal Tevatron duty factor (20 seconds of beam each minute) and that our detectors can handle $100 \mathrm{kHz}$ of beam particles, one week of running (assume $50 \%$ accelerator efficiency) will give us about $2 \times 10^{5} \quad \Sigma^{+} \rightarrow p \gamma$ decays. So there are lots of our favorite decays available: we just need a way to identify them.

Let us enumerate what will be the number of decay we would expect for one second's worth of beam

$\begin{array}{ll}100,000 . & \text { total particles } \\ 2000 . & \text { total } \Sigma^{+} \\ 1030 . & \Sigma^{+} \rightarrow p \pi^{\circ} \\ 970 . & \Sigma^{+} \rightarrow n \pi^{+} \\ 2.4 & \Sigma^{+} \rightarrow \mathrm{D \gamma}\end{array}$

Let us look at the $1030 \Sigma^{+} \rightarrow p \pi^{\circ}$ events and see how their number could be reduced in the trigger. If we reject events where both lead glass detectors had a single hit, we would reduce this to 500 events per second. We feel this can be further reduced by a factor of ten with the on 
line requirements on the position of the photon (see Figures $5 a$ and $5 \mathrm{~b}$ ). We are thus left with a managable trigger rate from $\Sigma^{+} \rightarrow p \pi^{\circ}$ decays of 50 per second.

In Figure 10 we illustrate the clean separation we have for the two decays $\Sigma^{+} \rightarrow p \gamma$ and $\Sigma^{+} \rightarrow p \pi^{\circ}$ before any off line cuts are made. Here we assume that only one photon from the $\pi^{\circ}$ has been detected. Additional criteria on momentum balance (Figure 11) and proton missing mass will reduce the the $\Sigma^{+} \rightarrow p \pi^{\circ}$ contamination by another order of magnitude. What is more important is that these criteria completely eliminate background arising from $\Sigma^{+} \rightarrow p \pi^{\circ}$ decays outside of our fiducial volume. In the Monte Carlo sample sample of $5000 \Sigma^{+} \rightarrow p \gamma$ centered about the gamma missing mass, we estimate a background of less than $0.1 \%$.

With a $50 \%$ live time (in addition to an overall $50 \%$ accelerator efficiency) we would get $\sim 10^{5} \Sigma^{+} \rightarrow p \gamma$ events in a week. If we assume a somewhate pessimistic detection efficiency of $25 \%$ for these events, we would be able to record $\sim 25,000$ events per week. This is a very substantial improvement over the present world sample of about one hundred events.

How large an event sample do we need to measure $\alpha \gamma$ ? If we assume our $20 \%$ polarization and that $\alpha_{\gamma}=-0.70$ (the present world average) we will need 25000 events to get $\Delta \alpha_{\gamma}=0.055$.

\section{RATES: $\Xi^{-} \rightarrow \Sigma^{-} \gamma$.}

Again let us assume a total beam rate of $100 \mathrm{~K}$ particles per second and look at its composition

$$
\begin{array}{ll}
100,000 . & \text { total particles } \\
2000 . & \text { total } \Xi^{-} \\
720 . & \Xi^{-} \rightarrow \Lambda \pi^{-} ; \Lambda \rightarrow n \pi^{\circ}: \pi^{\circ} \rightarrow \gamma \gamma \\
\geq 0.34 & \Xi^{-} \rightarrow \Sigma^{-} \gamma
\end{array}
$$

We assume $a \Xi^{-} \rightarrow \Sigma^{-} \gamma$ branching ratio of $1.7 \times 10^{-4}$, which is the unitarity limit given by Kogan and Shifman (1983).

Again we are raced with reducing the trigger rate from the $\pi^{\circ}$ background by about an order of magnitude. We will use the same trigger requirements as for the $\Sigma^{+} \rightarrow p \gamma$ runs but here many of the background photons will originate further downstream (the $\Lambda$ must also decay). 
Scintillation counters to veto the low momentum $\pi^{-}$which must accompany the $\pi^{\circ}$ should also be effective to reduce this source of background at the trigger level. As stated previously, there is no kinematically similar state which would be a serious background in the off line analysis. With the same data taking assumption as for the $\Sigma^{+} \rightarrow p \gamma$ we should expect $\sim 3500$ events in a one week run. However, we feel that we will probably be penalized about another factor of ten because of the requirement that the $\Sigma^{-}$live sufficiently long that we measure its momentum. In that case we may be down to 300 events. However. recall that we have used the unitarity lower limit for the branching ratio.

Run Strategy And Data Taking. We feel this experiment will require about 600 hours of accelerator time. About 200 hours will be for the checkout of the new spectrometer (wire chambers and silicon strip detectors), photon detectors (lead glass and TRD), and the trigger system (including the on-line processor). We would expect to set up first on the $\Sigma^{+} \rightarrow p \gamma$ decay and when we have understood its trigger and background problems switch over briefly to $\Xi^{-} \rightarrow \Sigma^{-} \gamma$ where we would assess the possibly quite different backgrounds. We estimate that the data taking on each reaction will be 200 hours. 


\section{References}

Anmed, M. A. and Ross, G. G. Phys. Letters 59B (1975) 293.

Ankenbrandt, C. et al., Phys. Rev. Letters, 51 (1983) 863.

Bourquin, M. and Repellin, J. P., Phys. Reports 114 (1984) 100.

Cardello, T. R. et al., Fermilab-Pub-84/122-E, Submitted to Phys. Rev.

Chong-Huah, Lo, Phys. Rev. D26 (1982) 199.

Denisov, A. et al., Fermilab-Conf-84/134-E.

Eeg. J. O., University of Oslo Report 82-26, May 11,1982.

Farrar, G., Phys, Rev. D4 (1971) 212.

Gavroglu, K. and Gottlied, H. P. W., Nucl. Phys. B79 (1974) 168.

Gershwin, Lawrence K. et al., Phys. Rev. 188 (1969) 2077.

Gilman, F. J. and Hagelin, J. S., Phys. Lett. 133B (1979) 443.

Gilman, Frederick and Wise, Mark B., Phys. Rev. D19 (1979) 976.

Graham, R. H., and Pakvasa, S., Phys. Rev. 140 (1965) B1144.

Hara, Yasuo, Phys. Rev. Letters, 12 (1964) 378.

Heller, K. Review talk at the 1984 Spin Conference.

Holstein, B., Nuovo Cimento 2A (1971) 561.

Iso, C. and Kawaguchi, M., Prog. Theo. Phys. 16 (1956) 177.

Jastrzembski, E., Hyperon Fluxes at $800 \mathrm{GeV}$. Talk given at the Fermilab

Hyperon Workshop, Dec. 7-8, 1984 and Hyperon Note H-224.

Kawaguchi, M. and Nishijima, K., Prog. Theo. Phys. 15 (1956) 182.

Kogan, Ya. I. and Shifman, M. A., Sov. J. Nucl. Phys. 38 (1983) 628.

Manz, A. et al., Phys Lett. 96B (1980) 217.

Marshak, R. E. et al., Theory of Weak Interactions In Particle Theory,

Wiley-interscience, New York (1969) 600.

Particle Data Group, Rev. of Modern Phys. 56 (1984) Si.

Picek, I., Phys. Rev. D21 (1980) 3169.

Rameika, Regina A., Rutgers University Thesis, December 1981.

Rudaz, Serge, Talk at the Fermilab Hyperon Workshop, Dec.7-8, 1984.

Scadron, M. D. and Thebaud, L. R., Phys. Rev. D8 (1973) 2190.

Shifman, M. A. et al., Phys. Rev. D18 (1978) 2583.

Skovpen:, Yu. I. and Sushkov, O. P., Sov. J. Nucl. Phys. 34 (1981) 253.

Wilkinson, C. et al., Phys. Rev. Lett. 46 (1981) 803. 


\section{FIGURE 1}

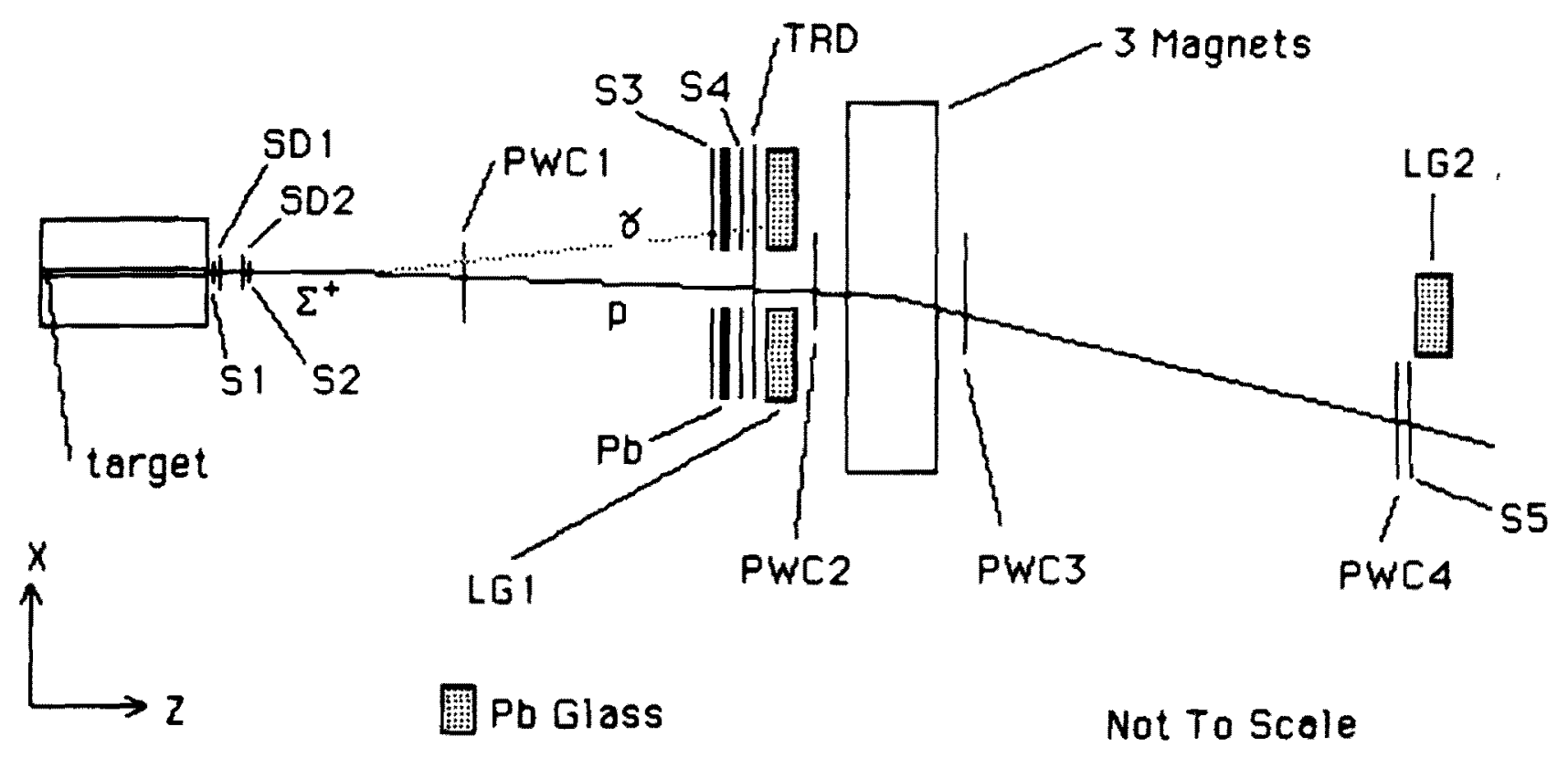

The Experimental Configuration 


\section{FIGURE 2}

\section{BEAM FRACTION at $10 \mathrm{~m}$}

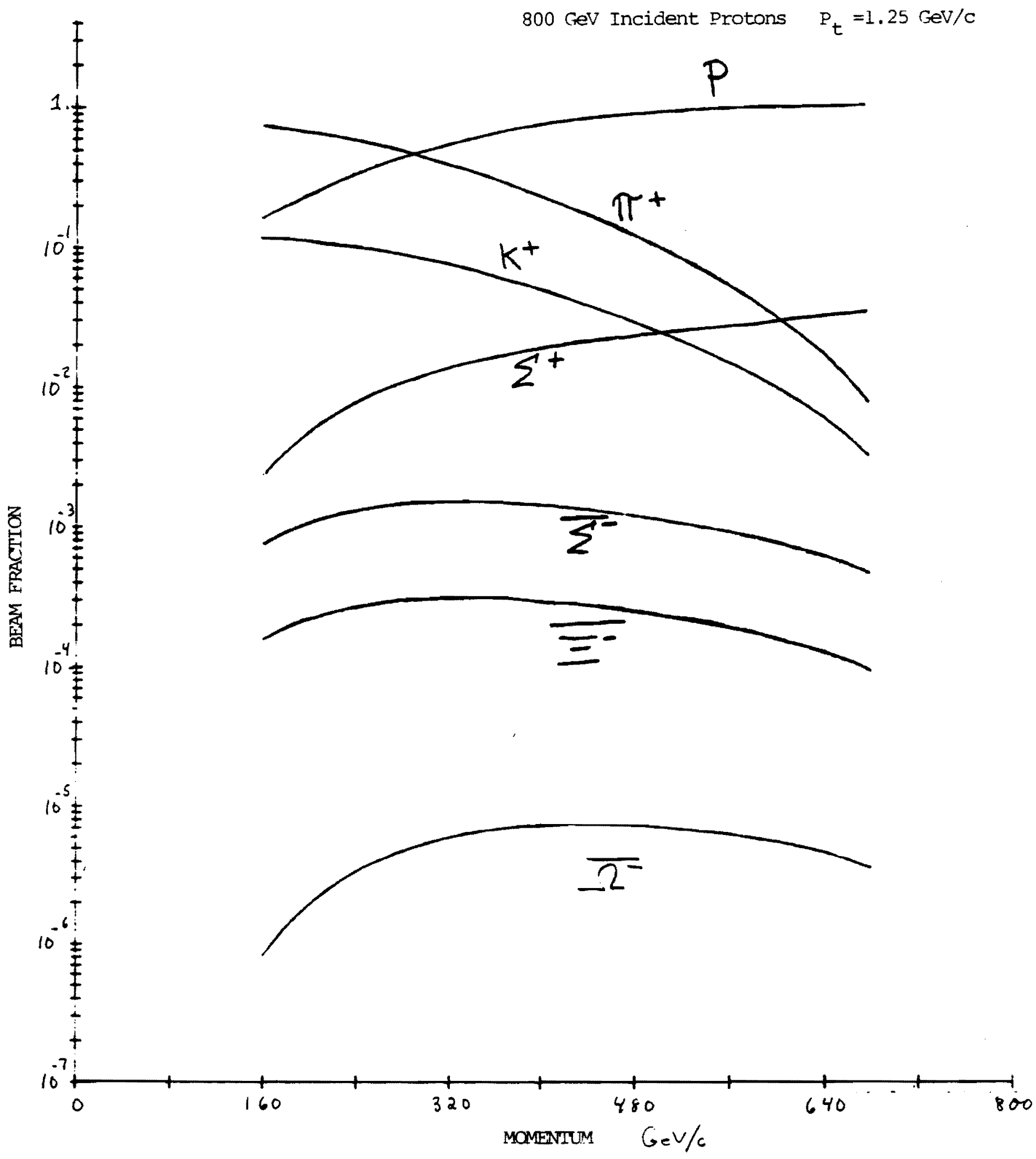


FIGURE 3

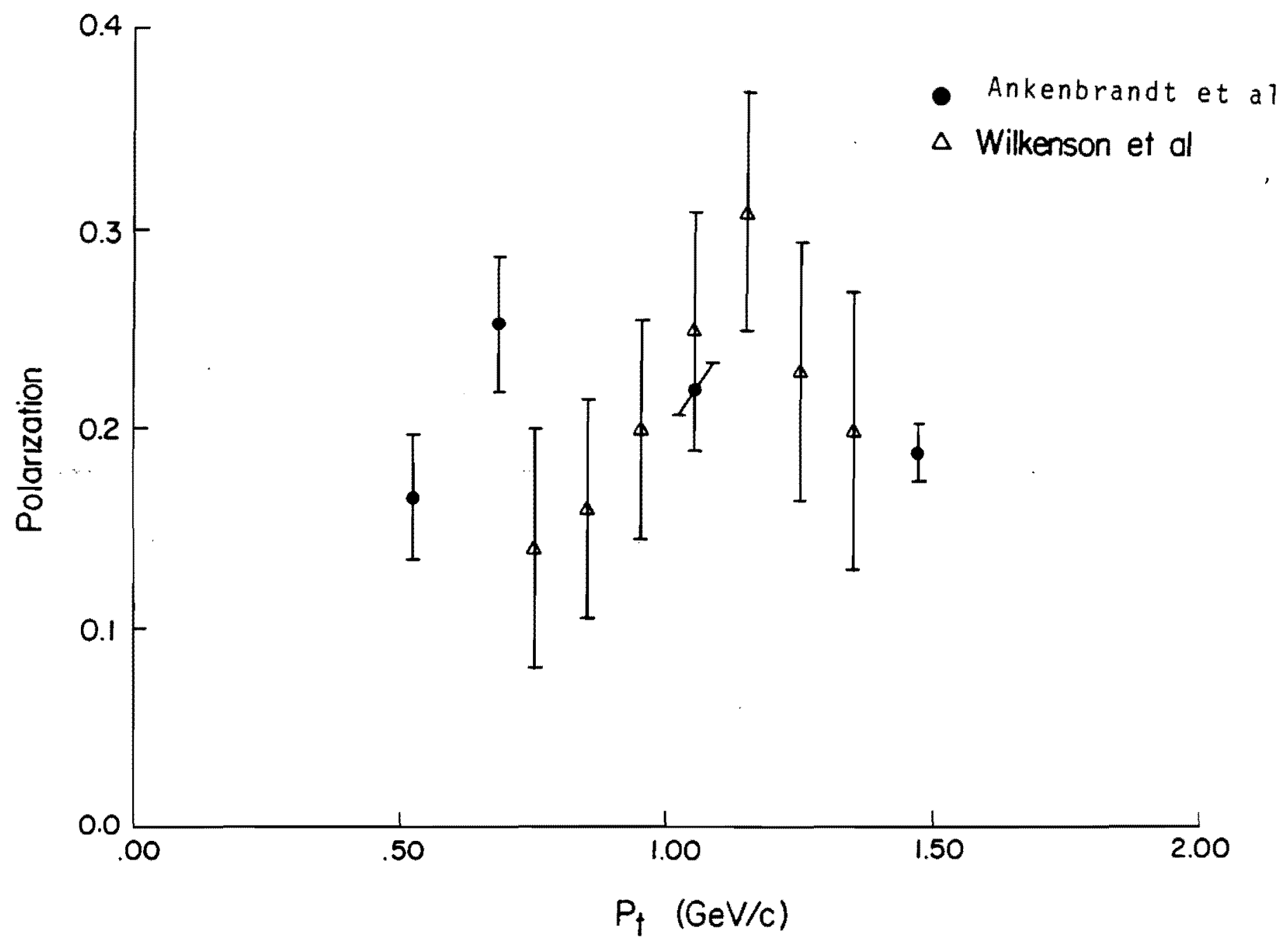

Polarization of $\Sigma^{+}$hyperons as a function of $P_{t}$ 


\section{FIGURE 4}

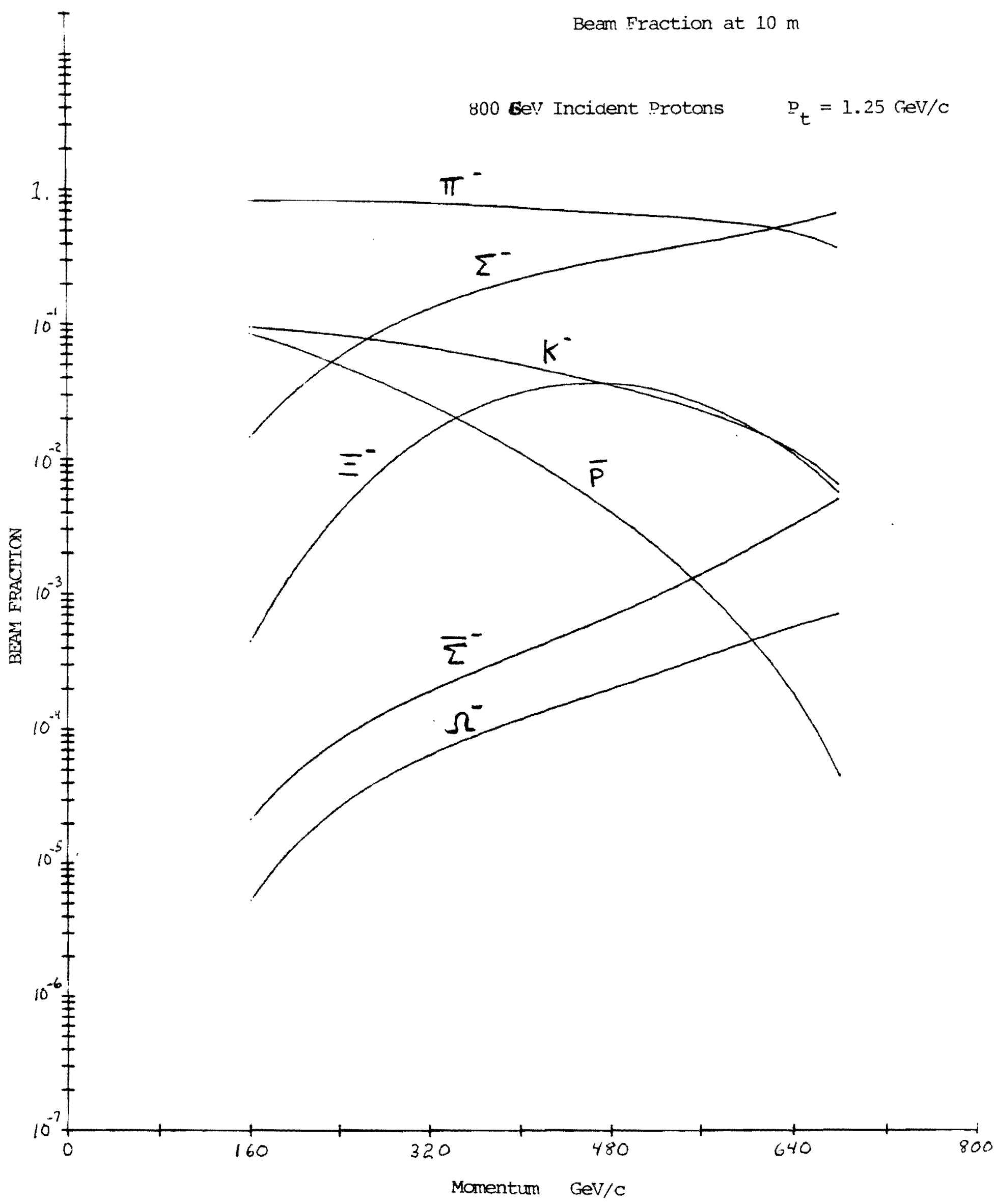


FIGURE 5

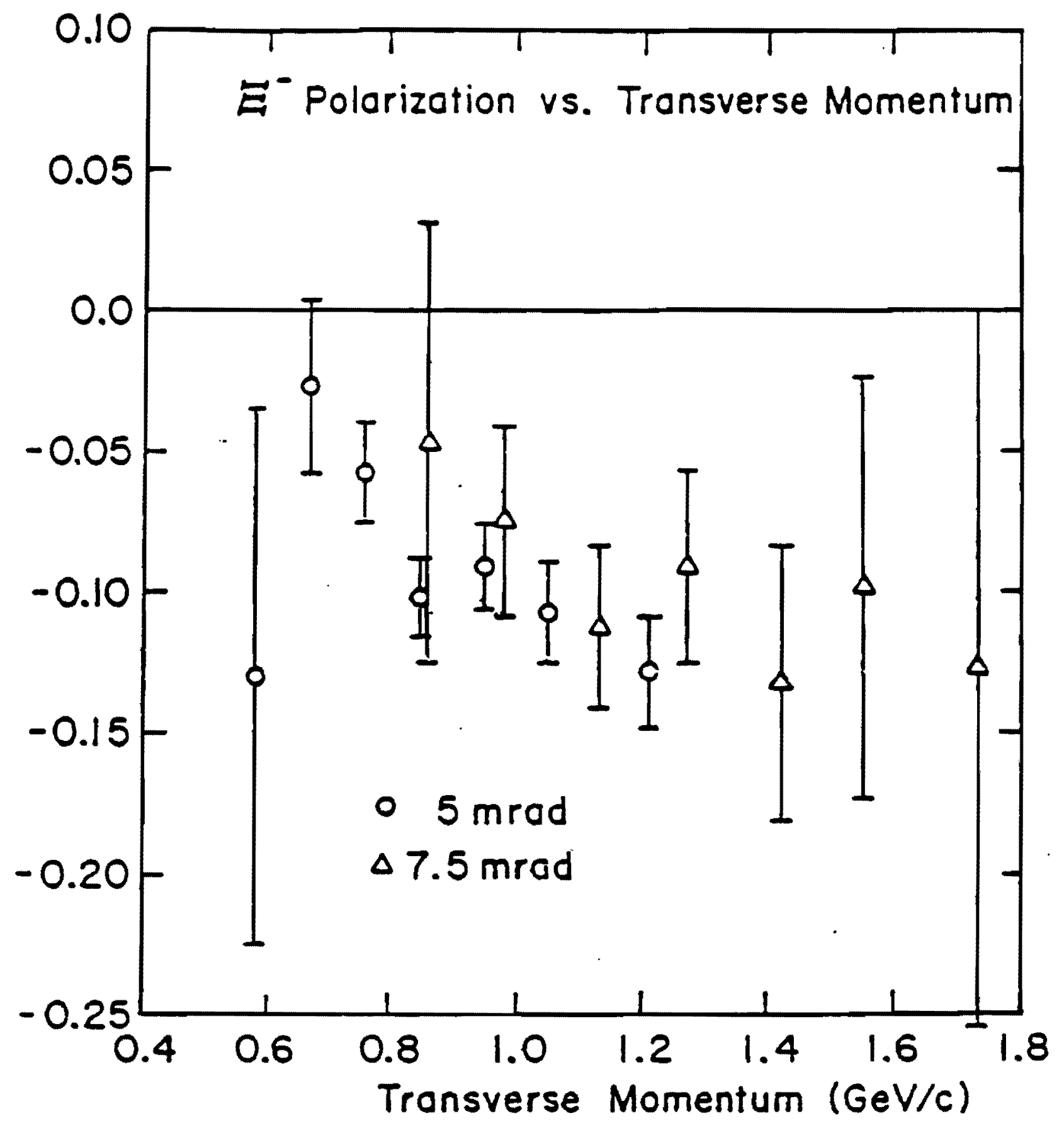



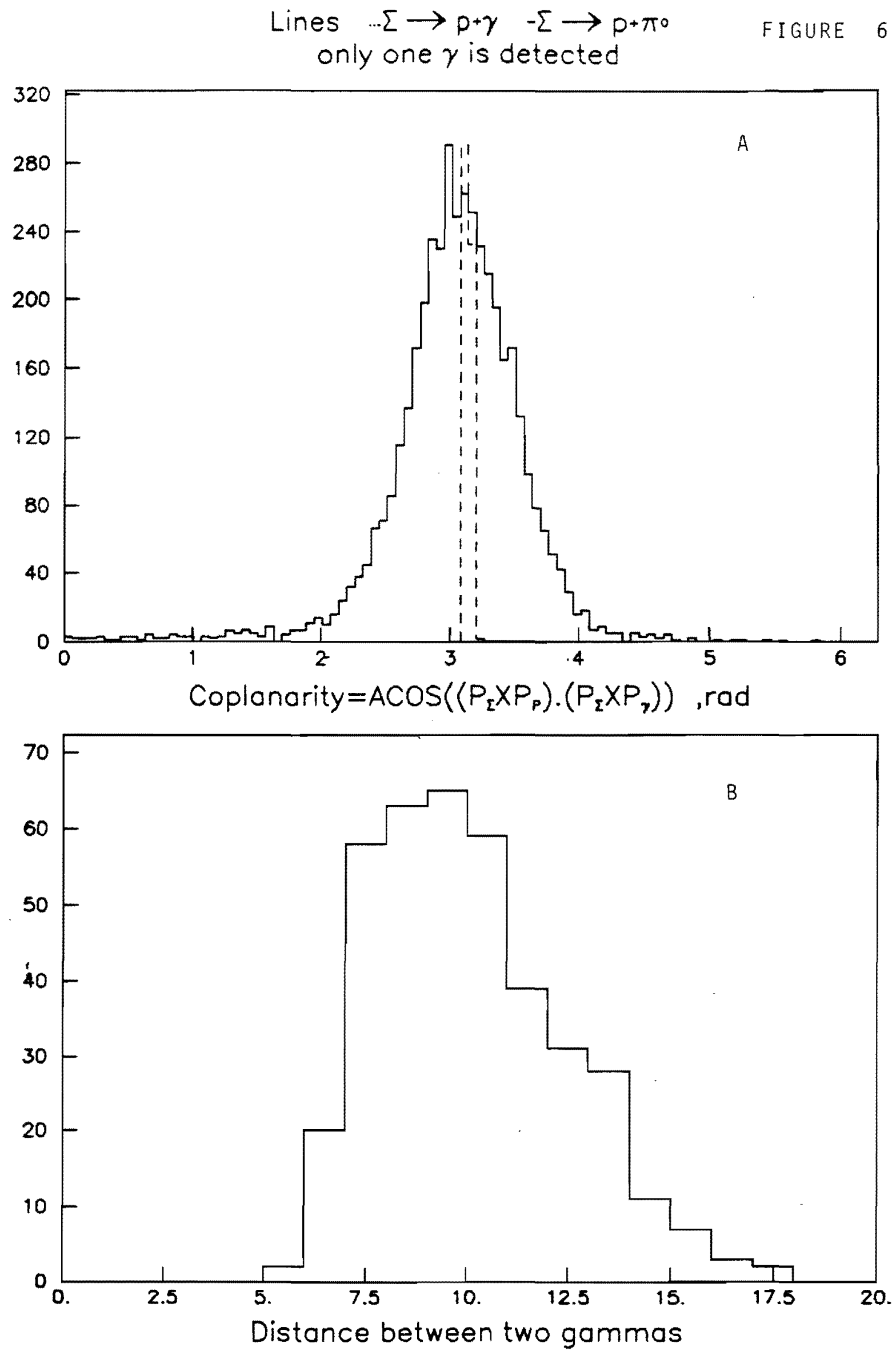


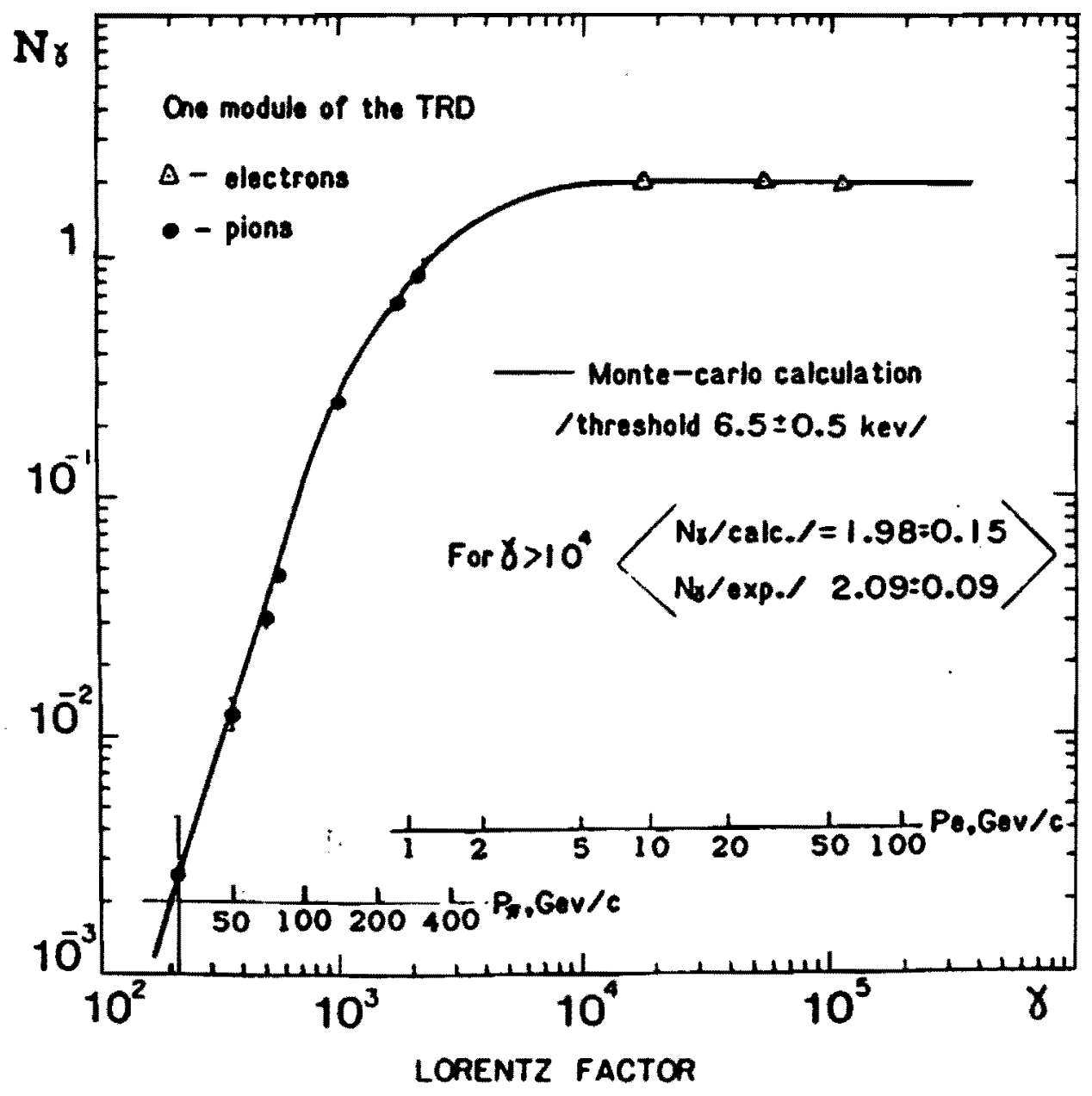


FIGURE 8

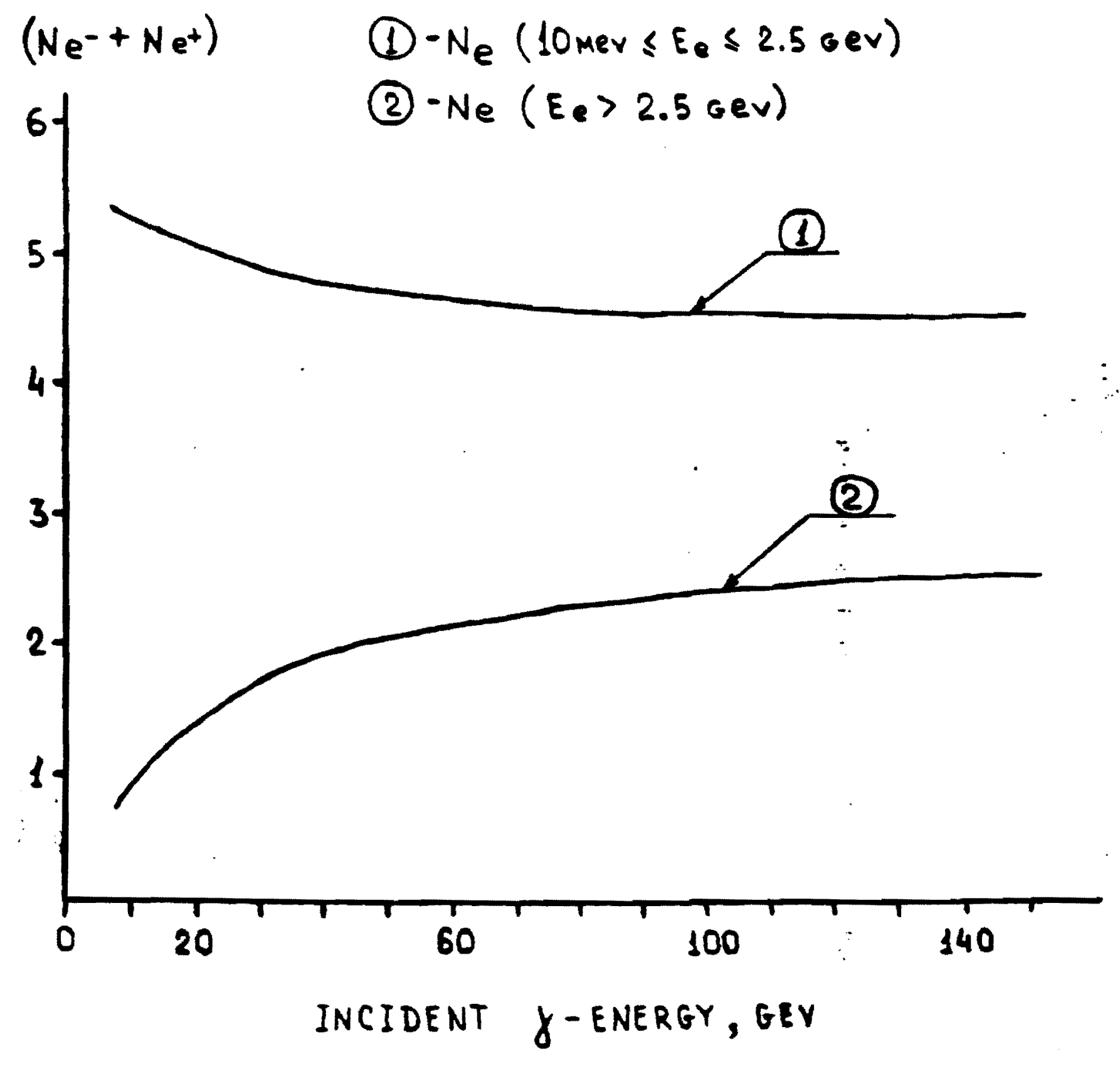


FIGURE 9

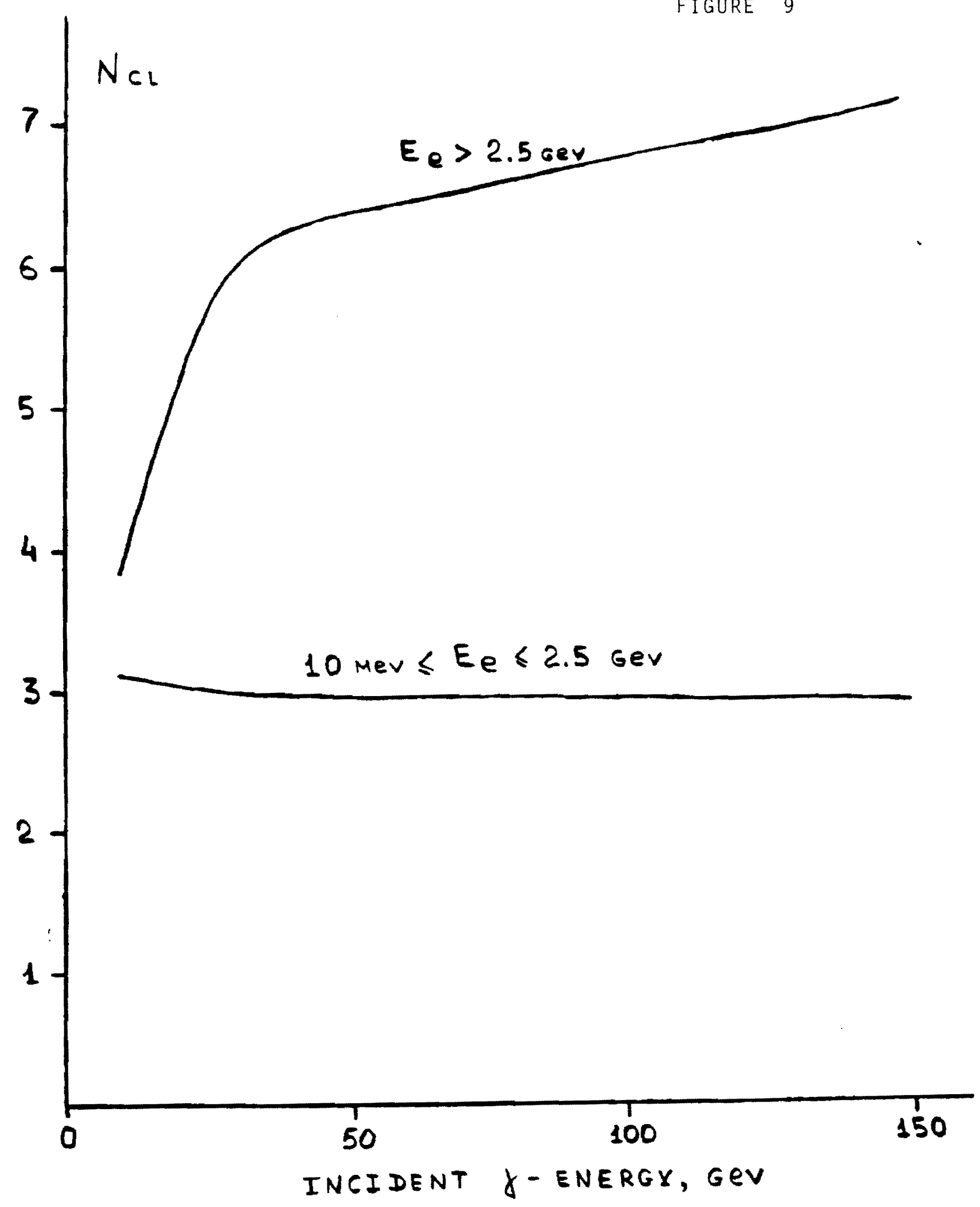


FIGURE 10

$$
\begin{gathered}
\left(P_{\Sigma}-P_{Y}\right)^{2} v s\left(P_{\Sigma}-P_{P}\right)^{2} \text { for } \Sigma \rightarrow p+\gamma \text { and } \Sigma \rightarrow p+\pi \circ \\
\text { only one } \gamma \text { is detected }
\end{gathered}
$$

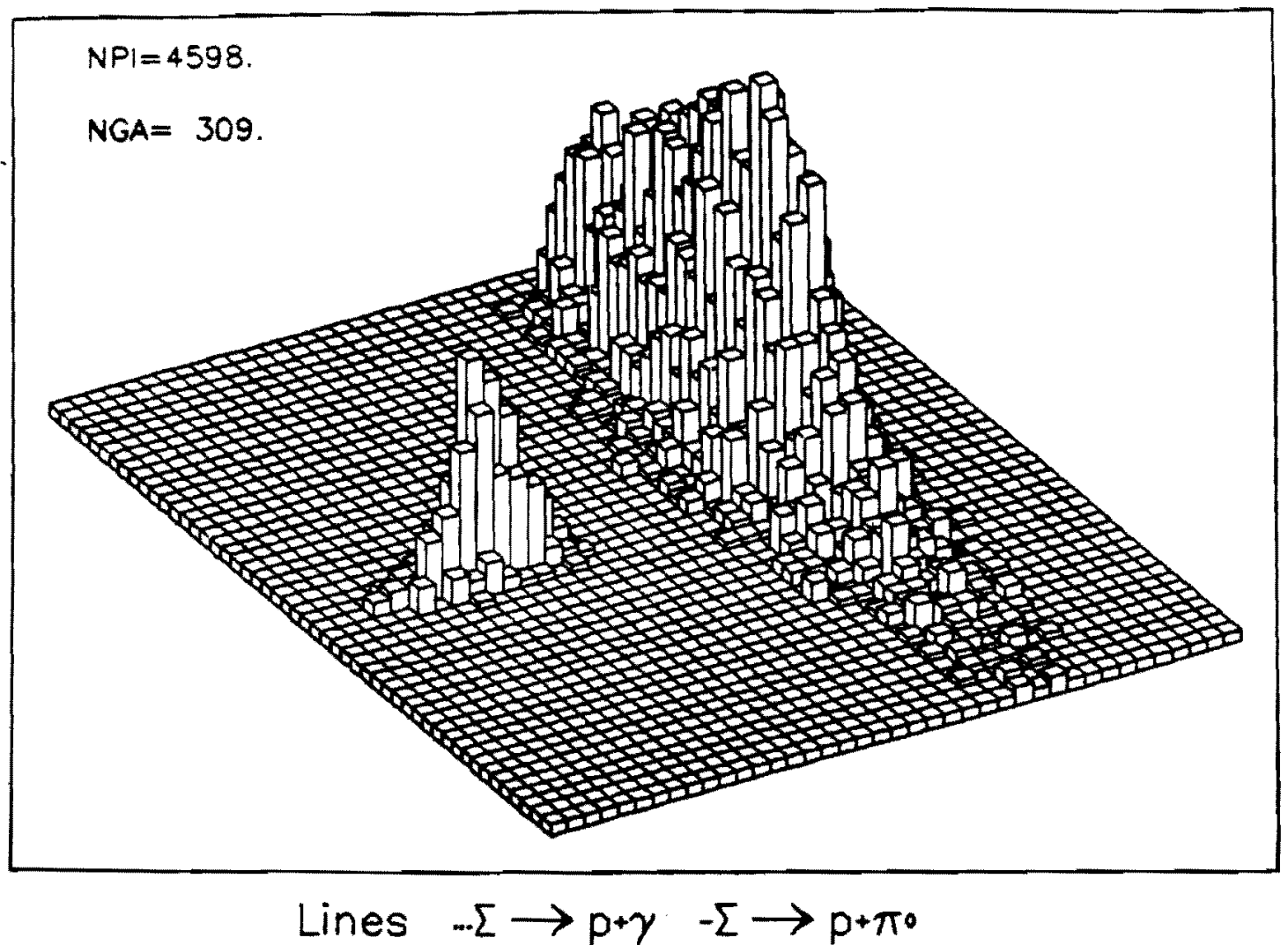

\section{$.10^{3}$}

$$
\text { Lines } \ldots \Sigma \rightarrow p+\gamma \quad-\Sigma \rightarrow p+\pi \cdot
$$
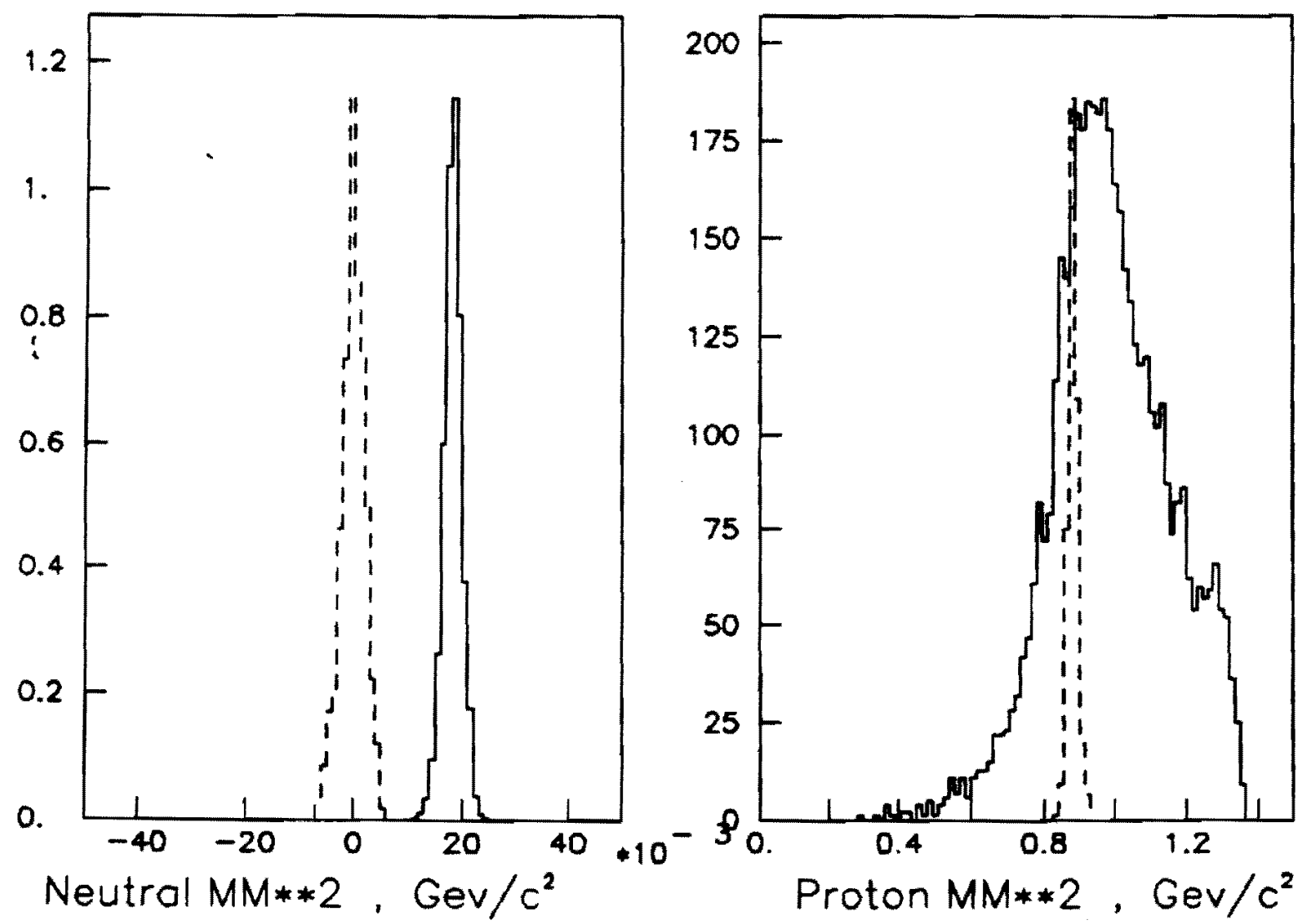
Lines $\quad \ldots \Sigma \rightarrow p+\gamma \quad-\Sigma \rightarrow p+\pi$ 。

only one $\gamma$ is detected
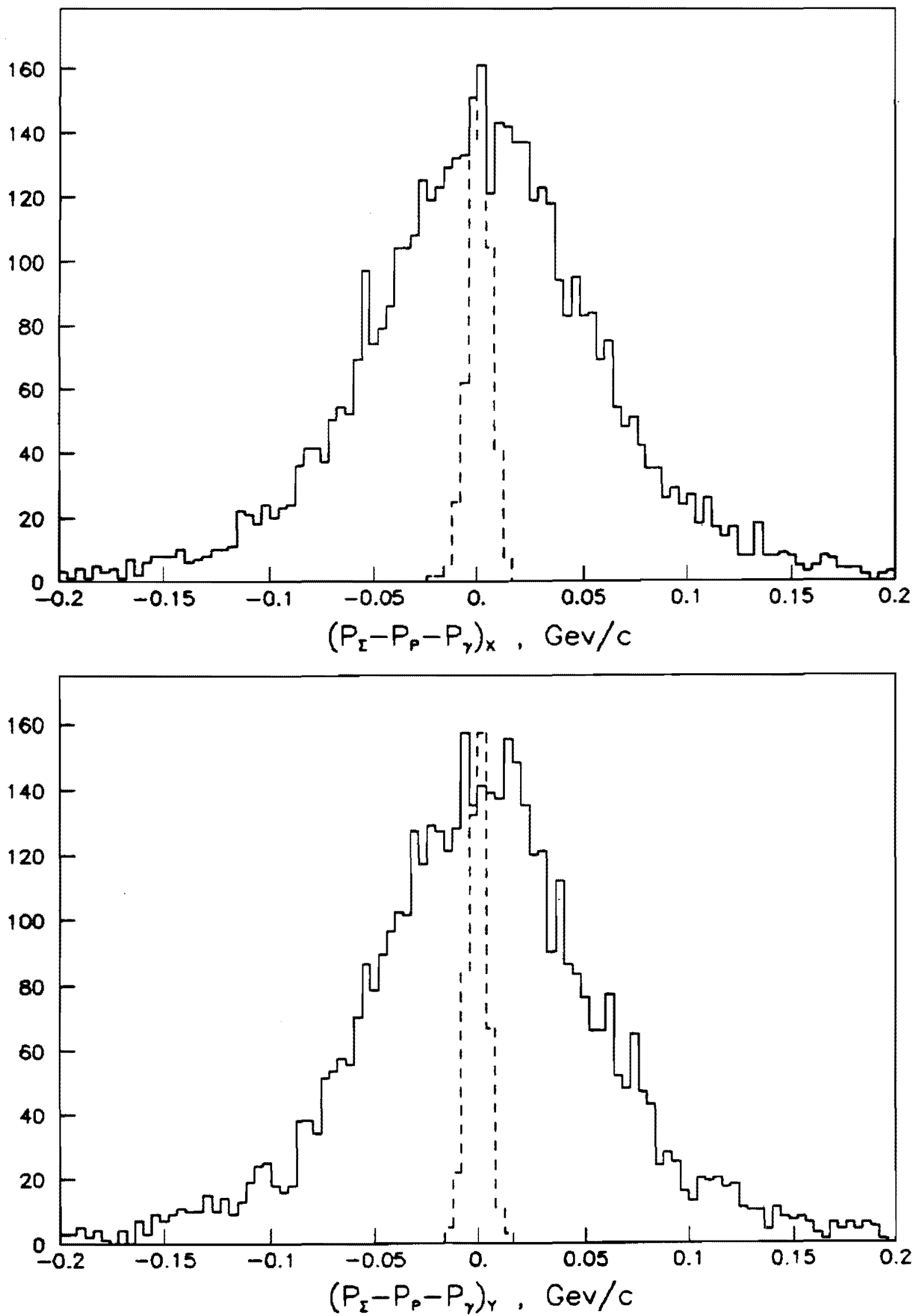


\section{An Electroweak Enigma: Hyperon Radiative Decays}

\section{$14 \rightarrow 25$}

\section{P. S. Cooper, J. Lach, A Marelos}

Fermilab

Batavia, Illinois 60510

Li Yunshan, Tang Fukun, Lang Fenglei. Li chengze

Institute of High Energy Physics

BeIjing. PAC

A. S. Denisov, V. Golovtsov, P Goriechev, V. Grachev. A Krivshich, N. Kuropatkin, V. Schegelsky. N. Smirnov, N. Terentyev, L. Uvarov. A vorcoyor.

Leningrad Nuclear Physics Institute

Leningrad, USSA

E. Mocliment, C. Newsom

University of lowa

lowa City. lowa 52242

C. Escooar. C. Golaman, P. Gouffon, J. Mation

University or São Paulo

Erazil

M. Foucher
Yale University

New Haven Comecticut 06511

Spckesmani A Vorcoyov:

Telephone: 2979143 Leningrad 
TLX-910-230-3233

IIRECTOR FNAL PROF L LEDERMAN

-ERHI NAT ACCELERATOR LAB /FNAL/

$>0 \mathrm{BOX} 500$

JATAVIA IL 60510 USA

JEAR LEON STOP UNFORTUNATELY I AM NOT ABLE TO COME TO THE PAC EETINO BUT I ASKED PETER COOPER AND YOE LACH TO PRESENT THE TATUS OF E 761 STOP

OU.WE ARE PRACTICALLY.BEADY TO START. THE EXPERIMENT STOP LL THE 32 MWPC TOOETHER UITH THE READ OUT ELECTRONICS FOR WELUE THOUSANDS CHANNELS HAVE BEEN PRODUCED IN GATTCHINA TESTED ND PREPARED FOR SHIPPING TO FINAL STOP ALSO THE TR GAMMA DETECTOR AS CONSTRUCTED AND TESTED IN GAMMA BEAMS AT

ERPUKHOU DEMONSTRATIHO THE SPACE RESOLUTION CLOSE TO THE EXPECTED TLUE STOP THE PREPARATION OF OTHER EQUIPMENT IN PARTICULAR 3D IS ALSO IN A BOOD SHAPE STOP SO THE E 761 COLLABORATION i EAGER TO START :THE EXPERIMENT AS SOON AS POSSIBLE STOP EANWHILE WE WISH GREAT SUCCESS TO THE CDF GROUP

HAVE ALREADY PROMISED TO MY STUDENTS THE DISCOUERY OF THE IP QUARK BY THE END OF THIS YEAR YOURS SINCERELY A VOROBYOU

L NIL

NN OALR OI APR 


\title{
An Electroweak Enigna: Hyporon Radiative Decays
}

\author{
E. Jastrzembeki, J. Lech, J. Morriner
}

Ferimileb

Batavia, Il!̣nols 60510

\section{Golovtsov, A. Krivshich, V. Schagplaty, R. Sminnow, \\ N. Torentyow, L. Uverow, A. Versoyov \\ Leningred Muclear Physics institute \\ Leningres, USSA}

E. McCliment, C. Newsom, E. Morbeck

University of lowa

lowa City, lows 52242

\author{
P. S. Cooper \\ Yale University \\ New Hoven, Comecticut 06511
}

Proposal Presentartoon: April 19, 1985

Spokesmani A Vorobyov

Telephone: 2979143 Leningrad 


$$
\Sigma^{+} \rightarrow p^{\gamma}, \equiv^{-} \rightarrow \Sigma^{-} \gamma \quad \text { Mensure } B R, \alpha_{\gamma}
$$

Experimental Data Meager

But significant progress since 1985

\section{HYPERON RADIATIYE DECAYS RII DWTL}

Branching Rat10 $\propto$ Events

\begin{tabular}{|c|c|c|c|c|}
\hline$\overline{\Sigma^{4} \rightarrow p \gamma}$ & $\begin{array}{l}1.22: 0.10 \times 10^{-3} \\
1.27: 0.17 \times 10^{-3} \\
1.30: 0.15 \times 10^{-3}\end{array}$ & $\begin{array}{l}-0.72: 0.29 \\
-0.86: 0.13: 0.04\end{array}$ & $\begin{array}{r}100 \\
155 \\
\frac{190}{190} \\
\end{array}$ & $\begin{array}{l}1986 \text { PDG } \\
\text { CERN, BIYI 1985 } \\
\text { Koosysni. 198? }\end{array}$ \\
\hline$\equiv^{-} \rightarrow \Sigma^{-} \gamma$ & $\begin{array}{l}<1.2 \times 10^{-3} \\
2.3 \times 1.0 \times 10^{-4}\end{array}$ & & 11. & $\begin{array}{l}1908 \text { PDG } \\
\text { CERK BIS1 198? }\end{array}$ \\
\hline$\Xi^{\bullet}-\wedge \gamma$ & $\begin{array}{l}5.0: 5.0 \times 10^{-3} \\
1.3 \pm 0.2 \times 10^{-3}\end{array}$ & & 170 & $\begin{array}{l}1986 \text { POG } \\
\text { umes, Fermlles E619 }\end{array}$ \\
\hline$\equiv \bullet-\Sigma^{\bullet} \gamma$ & $<7.0 \times 10^{-2}$ & & & 1986 PDG \\
\hline$\Lambda \rightarrow n \gamma$ & $1.02 \times 0.33 \times 10^{-3}$ & & 31 & CERH. BIY)I 1986. \\
\hline$\Omega^{-} \rightarrow E^{-} \gamma$ & $<2.2 \times 10^{-3}$ & & & 1986 PDG \\
\hline
\end{tabular}

Fermilab Hyperon Beam is unique facility to study these decays

Biagl et al. A measurement of the BR $\Sigma^{\circ}-D r / \Sigma^{\circ}-D \pi^{\circ}$. 2. Phys C28 (1985) $4955^{\circ}$." Blagl et al. First measurement of the $\wedge \rightarrow n \gamma$ BR. 2. Phys. C30 (1986) 201.

Biagi et al, First Masurement of the $\Xi^{-} \rightarrow \Sigma^{-} \gamma$ BR, 2. Phys. C35 (1987) 143. Kooayashi et al. New Measurement of the Asymmetry Parameter for the $\Sigma^{+} \rightarrow p r$ Decay. Ptys Rev Letters 59 (1987) 868

C. James. Seminar presented at fermllab. January 1986, H-302. 


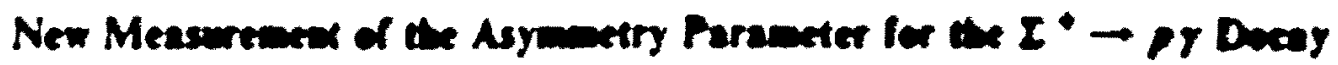

M. Kobayashi, (") J. Habe. "' T. Homma, ") H. Kawai. K. Miyake, T. S. Natomorz." (1) N. Saceo, and Y. Sugimoto(t)

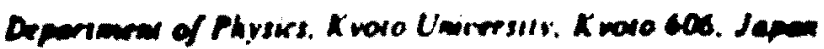

(1) 20 Man 1981)

De aymmetry parancter e, for the $I^{*}-p y$ decay has been measured with counter tecthiques.

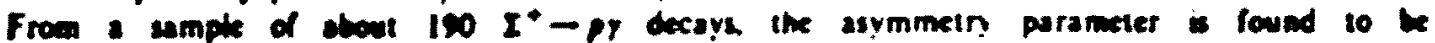
$0,--0.26 \pm 0.13 \pm 0.04$. where ine pwovd errors are salisical and sysuematix. respectively. The

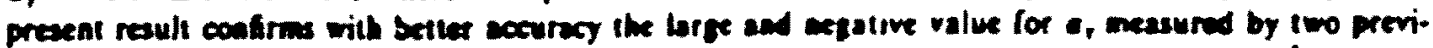

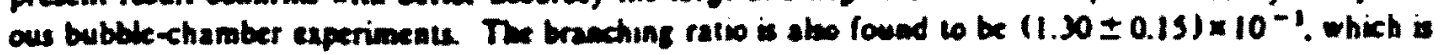

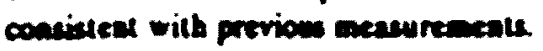

$$
\begin{aligned}
& \pi^{+}{ }_{f} \rightarrow R^{+} \Sigma^{+} \quad 1.76 .0 \mathrm{k} \\
& \leftrightarrow T^{\circ} \\
& \rightarrow ;
\end{aligned}
$$

$$
d_{2}=-0.81 \pm 0.12 \pm c .0
$$

\section{$E 92(\Sigma)$}

Meosurement of the Asymmetry

Poremeter in the I-PY Decoy.

Experimentol Selup .

(Side View)

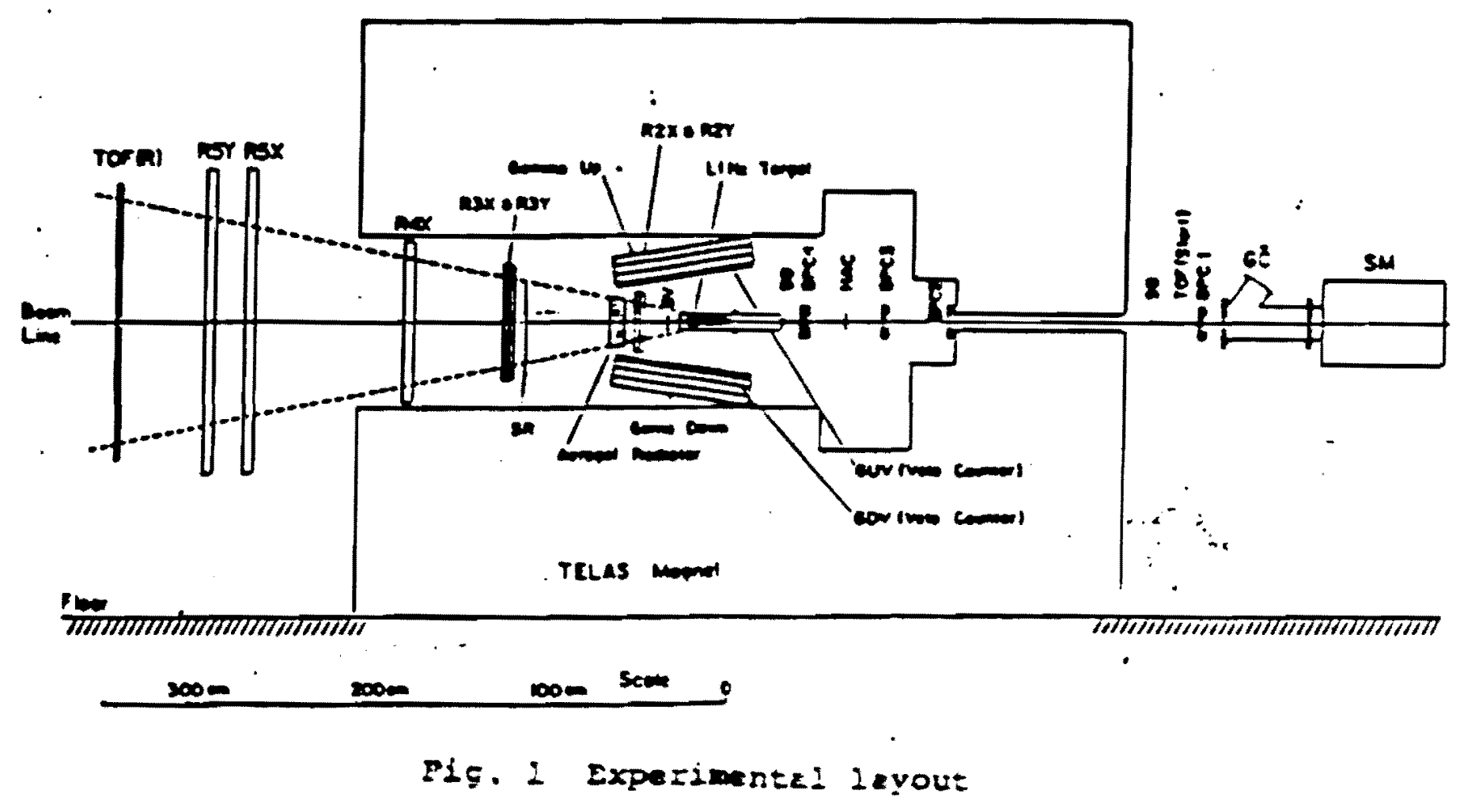


Single Quark Transition

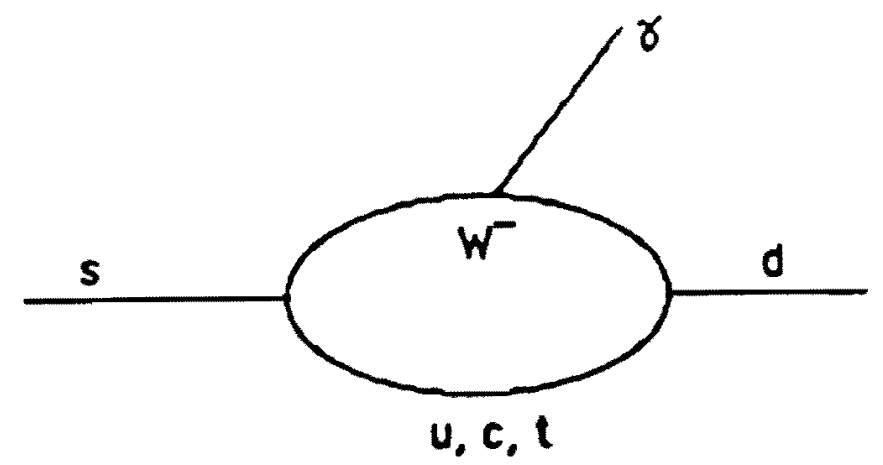

Rates are much too small

Gilman and Wise (1978) Assumed rate for $\Sigma^{+} \rightarrow p \gamma$

Predicted

$\Lambda \rightarrow n \gamma$

$\equiv-\rightarrow \Sigma-\gamma$

$\Xi^{\bullet} \rightarrow \Sigma^{\bullet} \gamma$

$\equiv \bullet \rightarrow \Lambda \gamma$

$\mathbf{Q}^{-} \rightarrow \Xi^{\circ} \gamma$
$2.2 \times 10^{-2}$

$1.1 \times 10^{-2}$

$8.1 \times 10^{-3}$

$4.0 \times 10^{-3}$

$4.1 \times 10^{-2}$
Measured

$1.02 \pm 0.33 \times 10^{-3}$

$2.3 \pm 1.0 \times 10^{-4}$

$<7.0 \times 10^{-2}$

$1.3 \pm 0.2 \times 10^{-3}$

$<2.2 \times 10^{-3}$

Kogan and Shifman (1983) The irk-hanism of this diagram cannot play an important role in weex radiative decays: 
Simplist Four Quark Diagram?

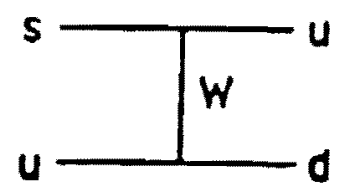

As Applied to to the decay $\Sigma^{+} \rightarrow p \gamma$

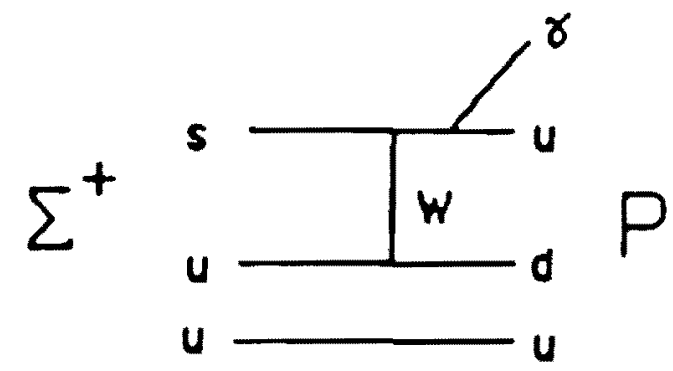

Can make substantial contribution to $\Sigma^{+} \rightarrow p \gamma$

but not to $\Xi^{-} \rightarrow \Sigma^{-} \gamma$ or $Q^{-} \rightarrow \Xi^{-} \gamma$

since they have to valence $u$ quarks 


\section{Penguin Diagrams}

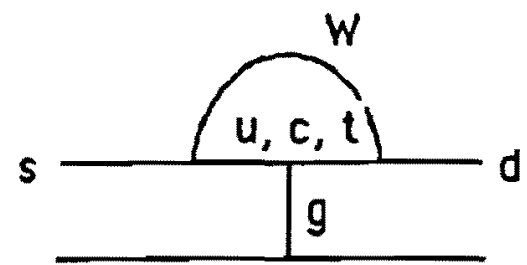

Schifman et al (1977)

Vainshtein et al (1978) Pointed out that the above may have large contributions not only to radiative decays but also

Should enhance $\Delta I=1 / 2$ decays

Contribute to $\varepsilon^{\prime}$ in $\varepsilon^{\prime} / \varepsilon$ of $K^{\circ} C P$ Violation 


\section{Asymmetries, $\alpha \gamma$}

Hara (1964) showed that $\alpha_{\gamma} \rightarrow 0$ if

Current-current interaction only left handed currents

CP invariance

$U$ spin symmetry

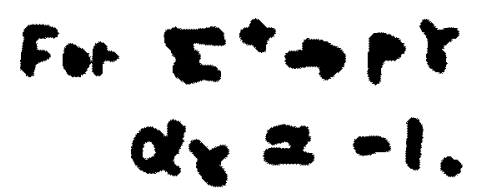

If relax $U$ spin symmetry requirement then in first order

$$
\begin{aligned}
\alpha_{\gamma} & =\left(m_{s}^{2}-m_{d}^{2}\right) /\left(m_{s}^{2}+m_{d}^{2}\right) \\
& \approx+0.5 \quad \text { Opposite to } \Sigma^{+} \text {measurements! }
\end{aligned}
$$

23 rears,

Recent paper by Li and LiU (CMU-HEP87-04) showed that Hara's theorem not true in general but is true for the decay $\Sigma^{+} \rightarrow p \gamma$ 
Decays $\Sigma^{+} \rightarrow p \gamma$ and $\Xi^{-} \rightarrow \Sigma^{-} \gamma$ probe different mechanisms so important to measure rate and $\alpha \gamma$ for each decay

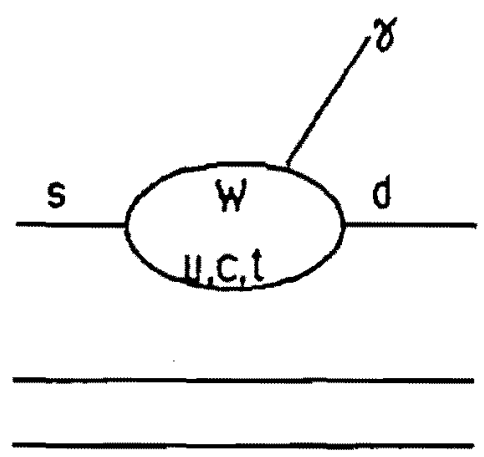

I Quark Transition

ALL

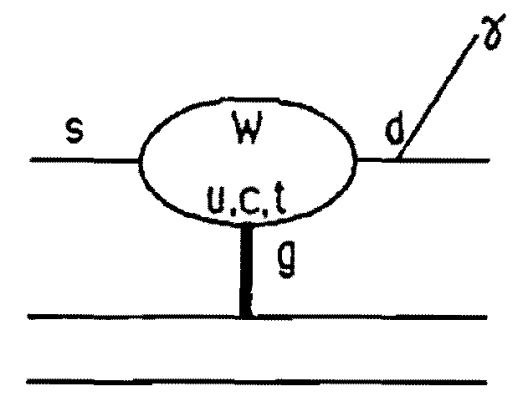

Penguin

ALL.

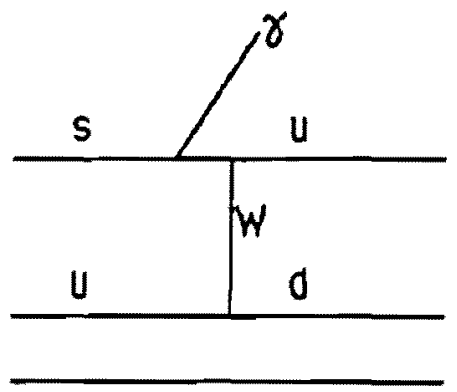

W Exchange

All Except

$$
\Xi^{-} \rightarrow \Sigma-\gamma
$$$$
\Omega^{-}+\Sigma^{-\gamma} \gamma
$$

Recent Contributions

Gaillard, Li, and Rudaz, Phys. Lett 18B (1985) Role of penguins, contributions from static color fields.

Balitskil. Brawn. and Kolesnichenko. Sov. J. Nucl. Phys. 44(6) 1986 QCD sum rules

Palle, Phys. Rev D36, (1987) 2863

Long distance effects 
$E 781^{\Sigma} \Sigma+p \gamma$

Aoril 3. 1 seo
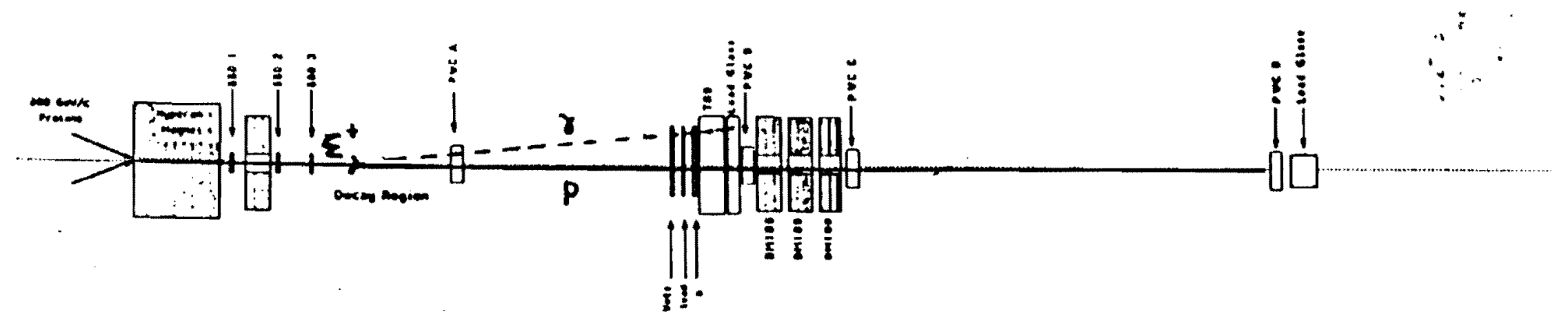

\section{$E 781 \cong \Sigma^{-} \gamma$}

ail 3, ins
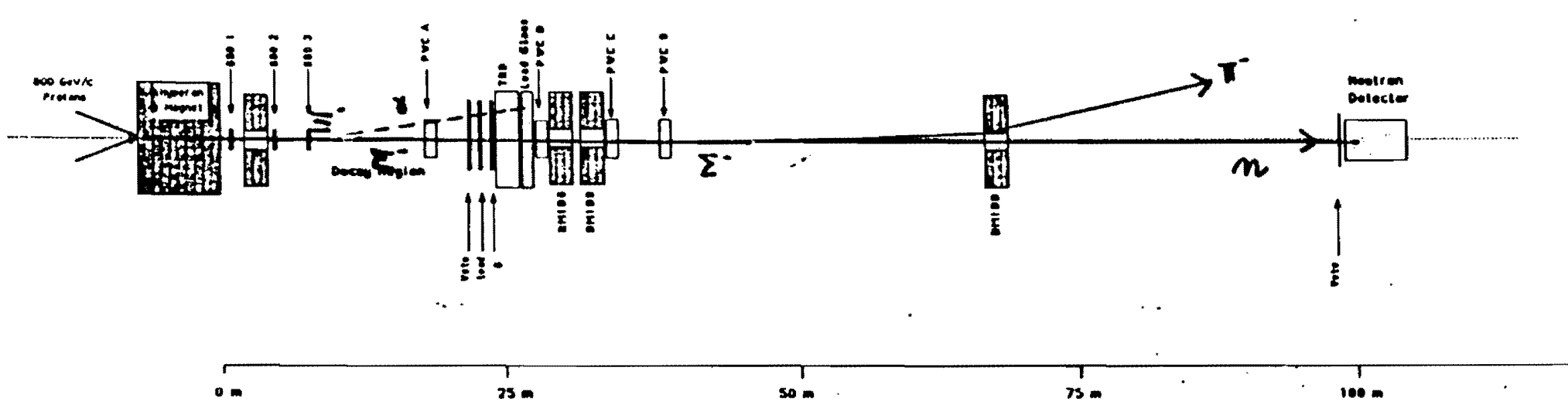


$$
\begin{aligned}
& \text { Fluxes } \quad 000 \mathrm{GeV} \text { Primary -.. } \\
& 350 \mathrm{GeV} / \mathrm{C} \text { Seconoary } \\
& 10 \mathrm{~m} \text { from targot } \\
& \text { - BEAM 100,000. total particles/sec } \\
& \text { 2000. total } \Sigma^{*} \\
& \text { 1030. } \Sigma^{*} \rightarrow p \pi^{\circ} \\
& \text { 970. } \Sigma^{*} \rightarrow \pi^{+} \\
& 2.4 \Sigma^{+} \rightarrow p \gamma \\
& \text { - BEAM 200,000. total particles/sec } \\
& \text { 4000. - total } \Xi^{-} \\
& \text {1440. } \Xi^{-} \rightarrow \wedge \pi^{-} \quad \Lambda \rightarrow n \pi^{\circ} \quad \pi^{\circ}+\gamma \gamma \\
& 1.0 \Xi^{-}+\Sigma^{-} \gamma
\end{aligned}
$$


E761 Measurements

We wish to measure both the branching ratio and gamma asymmetry in Sigma and Cascade radiative decay.

Sigma radiative decay

$$
\text { error }
$$

Branching ratio

$1 \%$ (stat) $<3 \%$

alpha parameter

$3 \%$

Cascade radiative decay

Branching ratio

alpha parameter

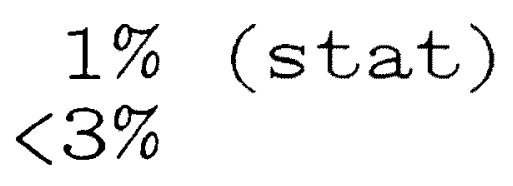

$10 \%$

events required

Sigma $\rightarrow$ P Gamma

$100 K$

Sigma $\rightarrow$ P Pizero

$1000 \mathrm{~K}$

Cascade -> Sigma Gamma

$30 \mathrm{~K}$

Cascade $\rightarrow$ Lamda Pizero 1000K 
E761 Status

Readout $\quad 12 \mathrm{~K}$ Channels built and Electronics tested in Leningrad. Controller Mods for noise in progress.

SSDs

Tested in $\mathrm{P}$-Center behind E756 during last run. $1000 / 5000$ channels tested Resolution $14 \mathrm{microns}$ Plane eff $95 \%$ Track eff $98 \%$
Noise
0.2-1 / plane

TRDs

Built in Leningrad

Tested at Serpukov

1100 channels

Resolution $1 \mathrm{~mm}$

Slight non-gaussian tails

PBG

Rutgers Glass (4 x 4 x 14) 72 Blocks

Currently in E756

Design in progress (EGS)

Needs restacking, stands. 
PWCs

Built in Leningrad

Tested in Leningrad

4500 Wires

NCAL

E715 Neutron Calorimeter Need only scintillators use only as counter Needs to be turned on

DAQ (old)

PDP 11/34 with Jorway 411 MULTI

Run during SSD tests

$1.4 \mathrm{msec} /$ trig deadtime Too Slow!

(new) E769 / E777 like system SCCs / RBUF / ACP nodes $0.15 \mathrm{msec} /$ trig deadtime Now being planned

Offline Software

Environment now running for SSD tests. 


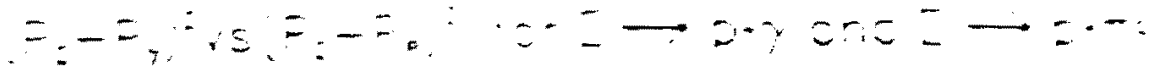

$$
\begin{aligned}
& \text { ony gre yis celectes }
\end{aligned}
$$

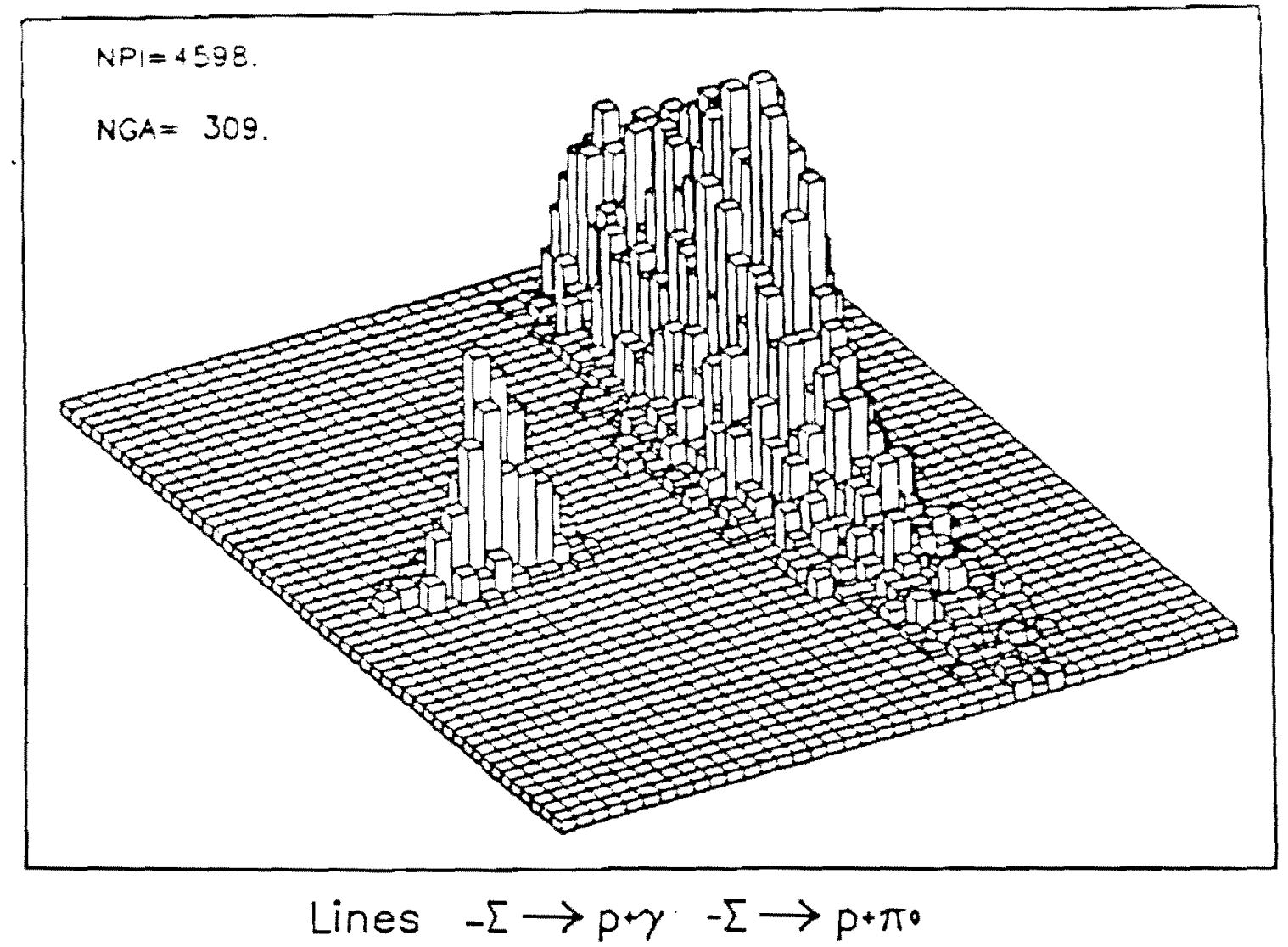

\section{$.10 \quad 3$}

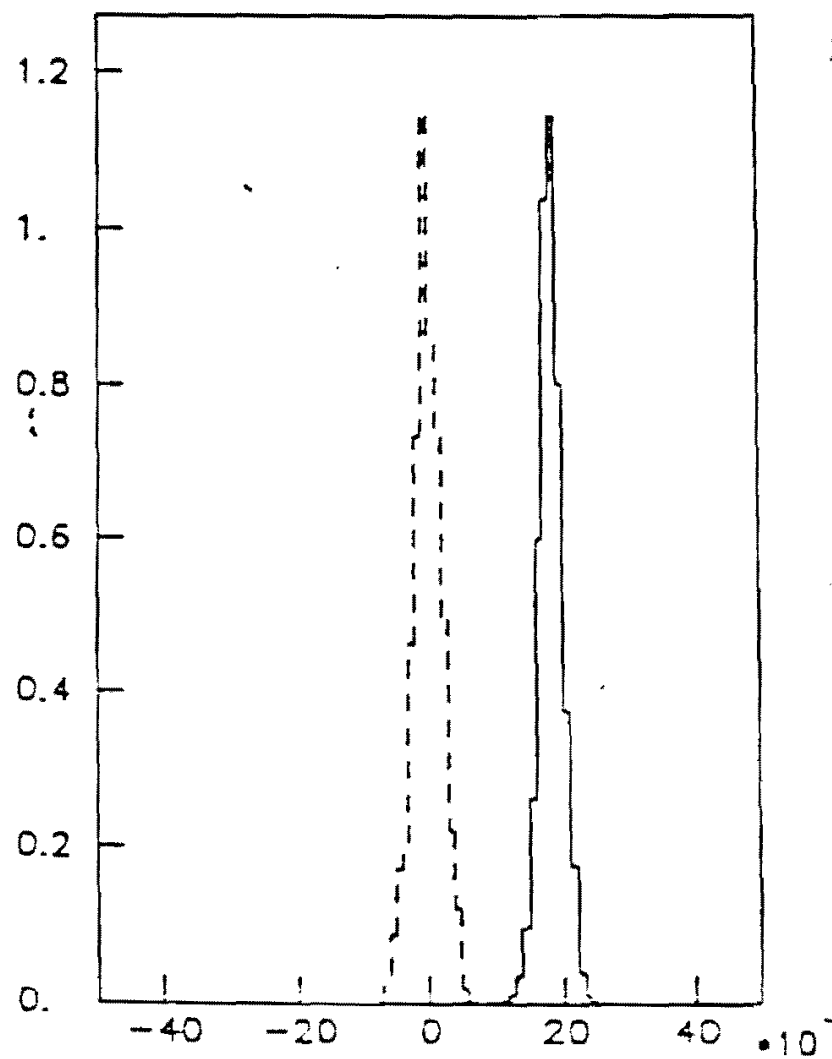

Neutrol MN*2, Gev/z=2

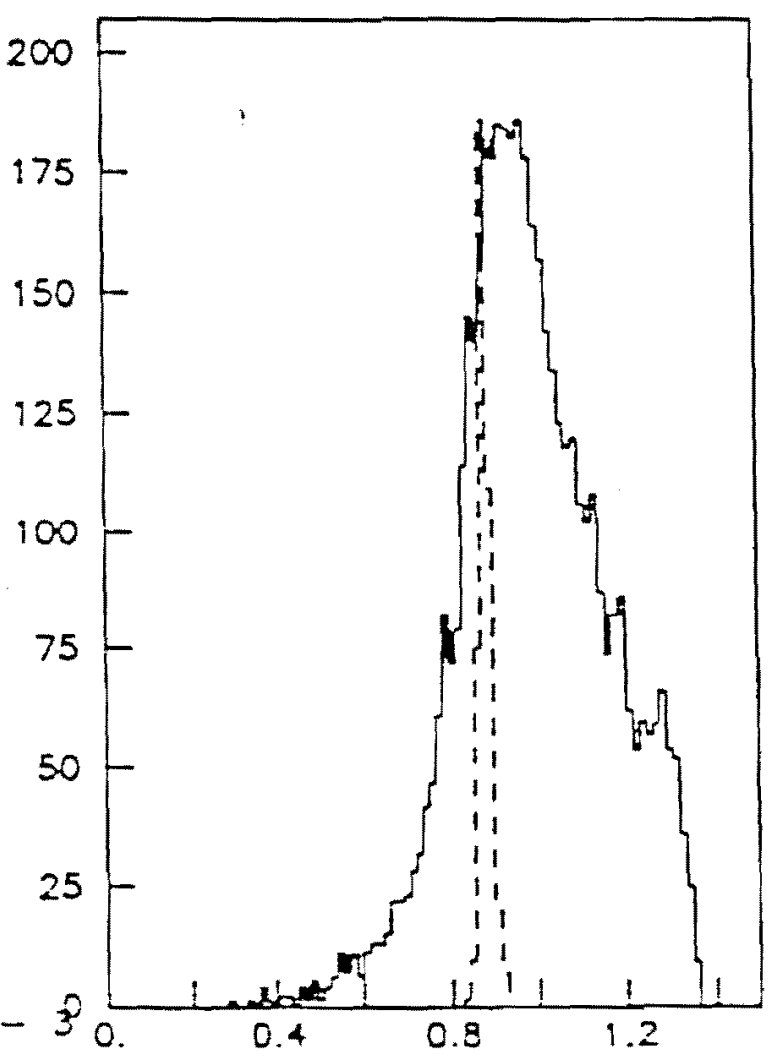

Proton Mik 2 , Gev/ $0^{2}$ 


\section{Experiment 777 DRTA RCquisition System}

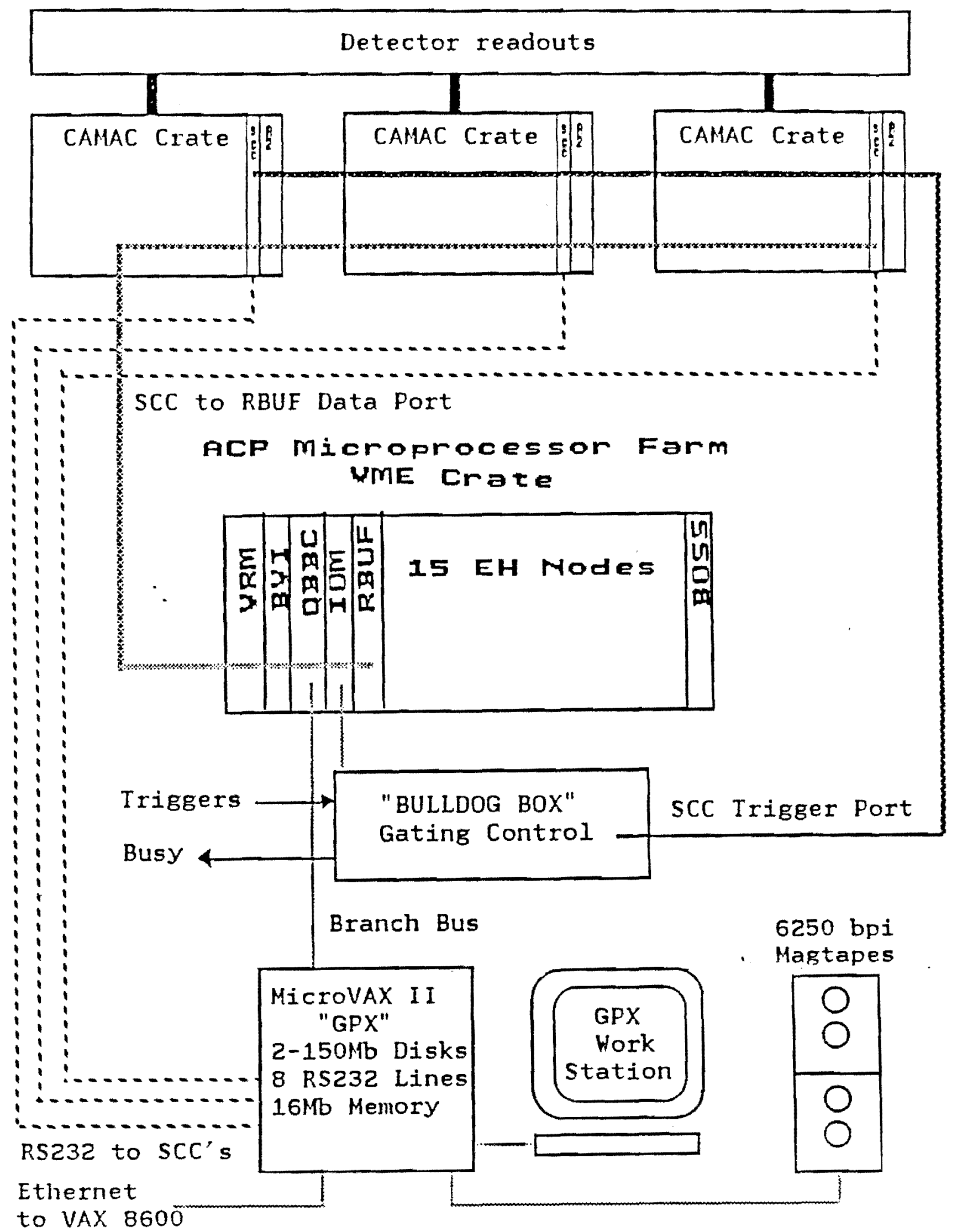


ET61 Rates

Beam

Sigma Cascade

Trigger

$100 \mathrm{khz} 200 \mathrm{khz}$

Livetime

$2 \mathrm{khz}$

$2 \mathrm{khz}$

Geometrical Eff

$70 \%$

$70 \%$

Reconstruction Eff $90 \%$

$90 \%$

Rate * BR

$2.4 \mathrm{hz}$

1. O hz

Good event rate

$0.6 \mathrm{hz} \quad .025 \mathrm{hz}$

Events/week

$60 \mathrm{~K}$

2500

weeks needed

2

12

Tapes (6250 bpi) $370 \quad 2200$

week : 20 sec/spill x 60 spills/hour $\mathrm{x} 83$ hours/week

$=100 \mathrm{~K}$ seconds of beam / week 
E 761 Requirements

* 2 - 4 months in P-Center 5/1/83 WITHOUT beam to build the $10 / 1 / 83$ experiment

* 2 months checkout with $11 / 1 / 83$

beam

$12 / 31 / 83$

* 3 months data taking

$1 / 1 / 84$

$2 / 14 / 84$

* Must be able to see the

signals while taking data

yes

* Answers online

yes

* All data on tape by the

end the next fixed target yes

run

* Public preliminary results

in about 6 months

$10 / 30 / 84$ 